\title{
Antiferromagnetic and Orbital Ordering on a Diamond Lattice Near Quantum Criticality
}

\author{
K. W. Plumb, ${ }^{1}$ J. R. Morey, ${ }^{1,2}$ J. A. Rodriguez-Rivera, ${ }^{3,4}$ Hui Wu, ${ }^{3}$ A. A. Podlesnyak, ${ }^{5}$ \\ T. M. McQueen, ${ }^{1,2,6}$ and C. L. Broholm ${ }^{1,3,5}$ \\ ${ }^{1}$ Institute for Quantum Matter and Department of Physics and Astronomy, The Johns Hopkins University, \\ Baltimore, Maryland 21218, USA \\ ${ }^{2}$ Department of Chemistry, The Johns Hopkins University, Baltimore, Maryland 21218, USA \\ ${ }^{3}$ NIST Center for Neutron Research, National Institute of Standards and Technology, \\ Gaithersburg, Maryland 20899, USA \\ ${ }^{4}$ Department of Materials Science and Engineering, University of Maryland, \\ College Park, Maryland 20742, USA \\ ${ }^{5}$ Quantum Condensed Matter Division, Oak Ridge National Laboratory, \\ Oak Ridge, Tennessee 37831-6473, USA \\ ${ }^{6}$ Department of Materials Science and Engineering, The Johns Hopkins University, \\ Baltimore, Maryland 21218, USA
}

(Received 29 March 2016; revised manuscript received 3 October 2016; published 16 December 2016)

We present neutron scattering measurements on powder samples of the spinel $\mathrm{FeSc}_{2} \mathrm{~S}_{4}$ that reveal a previously unobserved magnetic ordering transition occurring at 11.8(2) K. Magnetic ordering occurs subsequent to a subtle cubic-to-tetragonal structural transition that distorts Fe coordinating sulfur tetrahedra and lifts the orbital degeneracy. The orbital ordering is not truly long ranged, but occurs over finite-sized domains that limit magnetic correlation lengths. The application of $1 \mathrm{GPa}$ hydrostatic pressure appears to destabilize this Néel state, reducing the transition temperature to $8.6(8) \mathrm{K}$ and redistributing magnetic spectral weight to higher energies. The relative magnitudes of ordered $\langle m\rangle^{2}=3.1(2) \mu_{B}^{2}$ and fluctuating moments $\langle\delta m\rangle=13(1) \mu_{B}^{2}$ show that the magnetically ordered state of $\mathrm{FeSc}_{2} \mathrm{~S}_{4}$ is drastically renormalized and close to criticality.

DOI: 10.1103/PhysRevX.6.041055

\section{INTRODUCTION}

In a quantum spin liquid, quantum fluctuations overcome the development of any long-range magnetic order resulting in a dynamic state that breaks no symmetries at $T=0$. The search for this precarious state of matter in a real material is a decade's-old quest of condensed matter physics [1]. Geometrically frustrated magnetic materials are a focal point. Their competing exchange interactions can produce an extensive degeneracy that promotes quantum fluctuations and precludes the development of a staggered magnetization [2]. But spin frustrations is not the only mechanism to destabilize magnetic order. Orbital degeneracy that influences the spin sector through spinorbit coupling and impacts exchange interactions can enhance spin fluctuations and suppress magnetic order [3]. Orbital degrees of freedom may even form a quantum fluid in the presence of a staggered magnetization [4-6], or both spins and orbital sector can form a combined spinorbital liquid state $[7,8]$.

Published by the American Physical Society under the terms of the Creative Commons Attribution 3.0 License. Further distribution of this work must maintain attribution to the author(s) and the published article's title, journal citation, and DOI.
Subject Areas: Condensed Matter Physics, Magnetism, Strongly Correlated Materials
Spinel compounds, $A \mathrm{~B}_{2} X_{4}$, with magnetic ions occupying the $A$-site diamond sublattice form a simple, and therefore important, three-dimensional lattice with competing exchange interactions that frustrate the development of long-range magnetic order [9-13]. Amongst the $A$-site spinels, $\mathrm{FeSc}_{2} \mathrm{~S}_{4}$ holds a special place as the $\mathrm{Fe}^{2+}\left(3 d^{6}\right)$ ions have both spin and orbital degrees of freedom. The tetrahedral crystal field of the $A$-site environment splits the Fe $3 d$ manifold into a lower $e$ doublet and higher-energy $t_{2}$ triplet, from which Hund's coupling yields a high-spin $(S=2)$ configuration. Because a single hole occupies the $e$ doublet, $\mathrm{Fe}^{2+}$ is Jahn-Teller active, yet, surprisingly, there have been no reported observations of any structural distortion or magnetic ordering down to $50 \mathrm{mK}$. In particular, while the magnetic susceptibility exhibits Curie-Weiss-like behavior with $\theta_{\mathrm{CW}}=-45 \mathrm{~K}[14,15]$, there is no clear indication of a phase transition in the low-temperature susceptibility data and the specific heat exhibits only a broad peak centered at $8 \mathrm{~K}$ rather than a sharp anomaly typically associated with symmetry breaking. In accordance with this, neutron scattering measurements on polycrystalline samples have so far not provided evidence for magnetic ordering. The data show dispersive spin excitations emerging from momentum transfer 
corresponding to the cubic $Q=(100)$ position with an apparent excitation gap of $\sim 0.2 \mathrm{meV}$ [16].

Based on these experimental observations, it was proposed that $\mathrm{FeSc}_{2} \mathrm{~S}_{4}$ is a spin-orbital liquid arising from competition between on-site spin-orbit coupling and Kugel-Khomskii exchange [17] in the Fe diamond sublattice $[18,19]$. While spin and orbital ordering is favored by the intersite interactions, atomic spin-orbit coupling favors local spin-orbital singlets. Consequently, $\mathrm{FeSc}_{2} \mathrm{~S}_{4}$ is predicted to lie near the quantum critical point separating the spin-orbital singlet phase from a magnetically and orbitally ordered state. This hypothesis appears supported by other neutron measurements that show a shift of the low-energy magnetic scattering to higher energies upon application of a magnetic field [20]. Also, terahertz and farinfrared optical spectroscopy measurements have revealed low-energy $(4.5 \mathrm{meV})$ excitations consistent with a socalled spin orbiton [21-23], an excitation of entangled spin and orbital degrees of freedom. In this scenario, varying the magnetic exchange interactions relative to the spin-orbit coupling via the application of hydrostatic pressure might drive $\mathrm{FeSc}_{2} \mathrm{~S}_{4}$ through the quantum critical point to antiferromagnetism [18].

Here, we show that $\mathrm{FeSc}_{2} \mathrm{~S}_{4}$ is near but on the ordered side of this spin-orbital quantum critical point. Our measurements on a high-quality polycrystalline sample reveal that $\mathrm{FeSc}_{2} \mathrm{~S}_{4}$ undergoes a magnetic ordering transition near the broad maximum in the specific heat. Our data also indicate a subtle tetragonal, Jahn-Teller-like, distortion that lifts the orbital degeneracy at high temperatures so the lowtemperature state can be described in terms of a spin-only Hamiltonian. Rather than inducing order, the application of hydrostatic pressure of $1 \mathrm{GPa}$ reduces the magnetic ordering temperature and shifts inelastic spectral weight to higher energies, apparently destabilizing the ordered state. The Néel state in $\mathrm{FeSc}_{2} \mathrm{~S}_{4}$ is strongly renormalized with a sublattice magnetization that is reduced to $44(1) \%$ of the full saturation moment. Correspondingly, $40(2) \%$ of the inelastic spectral weight is associated with non-spin-wave fluctuations. In conjunction, these results show $\mathrm{FeSc}_{2} \mathrm{~S}_{4}$ is magnetically ordered, but in close proximity to a spinorbital quantum critical point.

\section{METHODS}

Powder samples of $\mathrm{FeSc}_{2} \mathrm{~S}_{4}$ are synthesized by solidstate reaction of elemental $\mathrm{Fe}, \mathrm{Sc}$, and $\mathrm{S}$ in an evacuated quartz tube. Residual oxygen contamination is minimized through the use of high-purity elemental materials, including vacuum remelted $\mathrm{Fe}$ and distilled dendritic Sc, while maintaining strict air-free synthesis conditions. Details of the sample synthesis are contained in Ref. [24]. The sample is initially characterized by powder x-ray diffraction at $300 \mathrm{~K}$ using a $\mathrm{Cu}-K \alpha$ x-ray source. Additional highresolution synchrotron $\mathrm{x}$-ray diffraction measurements are acquired at $T=100 \mathrm{~K}$ using the powder diffractometer
11-BM located at the Advanced Photon Source as described in Appendix A 1.

Specific heat and bulk magnetization measurements are carried out on a cold-pressed pellet of $\mathrm{FeSc}_{2} \mathrm{~S}_{4}$ using a Quantum Designs PPMS. The MACS spectrometer at the NIST center for Neutron Research is used for neutron scattering measurements. For measurements at ambient pressures, the $0.8 \mathrm{~g}$ sample of $\mathrm{FeSc}_{2} \mathrm{~S}_{4}$ is mounted in an $\mathrm{Al}$ sample can. Measurements under hydrostatic pressure are conducted with the sample loaded into a stainless steel pressure cell and pressurized to $1 \mathrm{GPa}$ with $\mathrm{He}$ gas. The lattice parameter of a pyrolytic graphite standard loaded into the pressure cell immediately above the sample is used to monitor the quasihydrostatic pressure at low temperature. MACS was operated with a fixed final neutron energy of either 3.7 or $2.4 \mathrm{meV}$ with appropriate combinations of $\mathrm{Be}$ and $\mathrm{BeO}$ filters before and after the sample to suppress higher-order neutron contamination. No incident beam filter is used for measurements with incident energies above $5 \mathrm{meV}$, and data for these energies are corrected to account for contributions to the monitor count rate from higher-order neutrons [25]. Data collected in the neutron attenuating pressure cell are corrected for the measured energy-dependent neutron transmission of the cell. Neutron diffraction measurements on the same sample are performed on BT-1 at NIST utilizing a $\mathrm{Cu}(311)$ monochromator, neutron wavelength $1.54 \AA$, and $60^{\prime}$ collimation before the monochromator. A set of high-resolution neutron scattering measurements are conducted on the CNCS spectrometer located at the Spallation Neutron Source at Oak Ridge National Lab [26]. For these measurements, a separate $0.45-\mathrm{g}$ powder sample is prepared and CNCS is operated with a fixed incident neutron energy of $1.55 \mathrm{meV}$ and a resulting elastic energy resolution of $0.034 \mathrm{meV}$ (FWHM). For all scattering measurements background signal contributions from the sample environment are subtracted and signal count rates are converted to absolute values of the scattering cross section using the integrated intensity from nuclear Bragg reflections [27].

We explicitly define the normalized magnetic neutron scattering intensity as used in this work to enable accurate discussion of sum rules. The magnetic neutron scattering cross section for a powder sample as a function of momentum transfer $Q$ and energy transfer $E$ is expressed as

$$
\frac{d^{2} \sigma}{d \Omega d E^{\prime}}=N \frac{k_{f}}{k_{i}} r_{0}^{2} e^{-2 W(Q)}\left|\frac{g}{2} f(Q)\right|^{2} 2 \tilde{\mathcal{S}}(Q, E),
$$

where $N$ is the number of iron atoms, $k_{i}$ and $k_{f}$ are the incident and final neutron wave vectors, respectively, $r_{0}=$ $0.539 \times 10^{-12} \mathrm{~cm}$ is the magnetic scattering length, and $e^{-2 W(Q)}$ is the Debye-Waller factor, which we set to unity for low temperature and low $Q$. The spherically averaged dynamic structure factor is given by 
$\tilde{\mathcal{S}}(Q, E)=\int \frac{d \Omega_{Q}}{4 \pi} \frac{1}{2} \sum_{\alpha \beta}\left(\delta_{\alpha \beta}-\hat{Q}_{\alpha} \hat{Q}_{\beta}\right) \mathcal{S}^{\alpha \beta}(\mathbf{Q}, E)$,

where $g$ is the Landé $g$ factor, $f(Q)$ is the magnetic form factor, and the dynamic structure factor is defined as

$$
\begin{aligned}
\mathcal{S}^{\alpha \beta}(\mathbf{Q}, E)= & \frac{1}{2 \pi \hbar} \int d t e^{-i E t / \hbar} \\
& \times \frac{1}{N} \sum_{\mathbf{r}, \mathbf{r}^{\prime}}\left\langle S_{\mathbf{r}}^{\alpha}(0) S_{\mathbf{r}^{\prime}}^{\beta}(t)\right\rangle e^{-i \mathbf{Q} \cdot\left(\mathbf{r}-\mathbf{r}^{\prime}\right)},
\end{aligned}
$$

where $S_{\mathbf{r}}^{\alpha}$ is the $\alpha$ component of the effective spin operator. In this work, we report the normalized inelastic neutron scattering intensity per Fe:

$$
\tilde{I}(Q, E)=\frac{1}{N} \frac{k_{i}}{k_{f}} \frac{d^{2} \sigma}{d \Omega d E^{\prime}} .
$$

Rietveld refinement of neutron and synchrotron diffraction data is carried out using the general structure analysis system (GSAS) complimented with the SARAh program for magnetic symmetry analysis [28].

\section{RESULTS}

Our main experimental finding is presented in Fig. 1(a), which displays a false-color map of the elastic neutron scattering cross section for $\mathrm{FeSc}_{2} \mathrm{~S}_{4}$ as a function of temperature $(T)$ and momentum transfer $(Q)$. While the most prominent features are two structural Bragg peaks at $Q=$ 1.03 and $1.69 \AA^{-1}$ that saturate the color scale and are independent of temperature below $20 \mathrm{~K}$, the high-sensitivity measurement also reveals five temperature-dependent Bragg reflections located at $0.6,0.84,1.33,1.46$, and $1.77 \AA^{-1}$. Not previously reported, these peaks emerge in unison below $12 \mathrm{~K}$. At $T=1.6 \mathrm{~K}$, the peak intensity of the strongest magnetic Bragg peak at $Q=0.6 \AA^{-1}$ amounts to $20 \%$ of the strongest nuclear Bragg peak intensity at $Q=1.69 \AA^{-1}$. In the following, we argue that these Bragg peaks arise from a magnetic ordering transition that is enabled by a higher-temperature orbital ordering transition. Details of the lowest $Q$ magnetic Bragg reflection are shown in Figs. 1(b) and 1(c). We fit the high-resolution data using a Lorentzian profile convolved with a Gaussian instrumental resolution of $0.045 \AA^{-1}$ FWHM. This analysis reveals an intrinsic Lorentzian half width at half maximum of $\kappa=0.019(6) \AA^{-1}$, which corresponds to a correlation length of $\xi=\kappa^{-1}=53(16) \AA$. Similarly, the energy dependence of the $Q$-integrated peak is fit to a quasielastic Lorentzian profile convolved with the Gaussian instrumental resolution to yield a lower bound on the correlation time of $\tau>0.4 \mathrm{~ns}$. We argue below that the finite magnetic correlation length is a result of exchange disorder built into the system at the higher-temperature orbital occupational ordering transition. The absence of
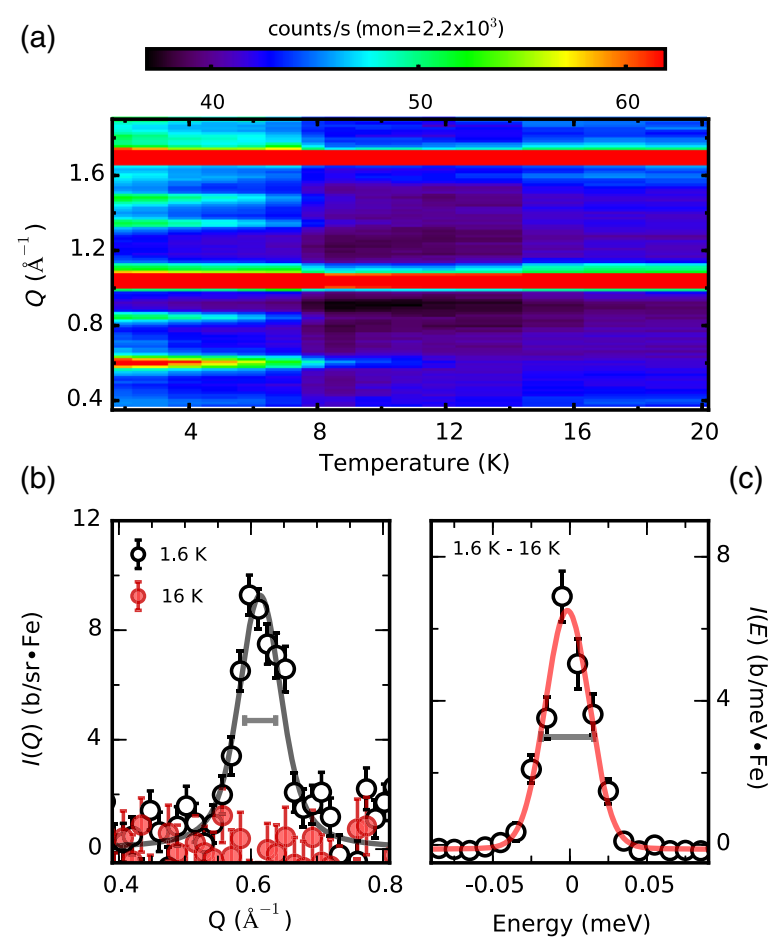

FIG. 1. (a) Overview of the temperature-dependent neutron diffraction intensity in $\mathrm{FeSc}_{2} \mathrm{~S}_{4}$ as measured on MACS. Data were acquired for a fixed monitor count (mon). (b) Magnetic Bragg reflection measured on CNCS integrated between $-0.018<$ $E<0.018 \mathrm{meV}$. An identical constant background has been subtracted from low- and high-temperature data sets. (c) Energy dependence of magnetic Bragg reflection integrated over $0.52<Q<0.72 \AA^{-1}$. Horizontal bars in (b) and (c) indicate the instrumental resolution as determined from corresponding cuts through the nuclear (111) Bragg peak at $1.04 \AA^{-1}$. Error bars represent 1 standard deviation.

previous reports of magnetic order is consistent with an extreme sensitivity of the ground state of $\mathrm{FeSc}_{2} \mathrm{~S}_{4}$ to chemical and/or crystalline defects, as might be expected near quantum criticality.

\section{A. Bulk magnetization}

The temperature dependence of the bulk magnetic susceptibility of $\mathrm{FeSc}_{2} \mathrm{~S}_{4}$ is shown in Fig. 2. The hightemperature susceptibility displays Curie-Weiss behavior with a paramagnetic moment of 5.1(1) $\mu_{B}$ and Curie-Weiss temperature $\theta_{\mathrm{CW}}=-40.1(5) \mathrm{K}$, fully consistent with earlier reports [14]. However, at lower temperatures our data exhibit a broad turnover at $\sim 10 \mathrm{~K}$, where previous reports have a strong Curie tail [14]. The turnover observed in our samples is identical to the local susceptibility extracted from Sc nuclear magnetic resonance (NMR) Knight shift measurements [15,29]. The NMR Knight shift probes the intrinsic local spin susceptibility, independent of any Curie tail contribution from small amounts of impurities that may dominate the bulk susceptibility at low temperatures. 


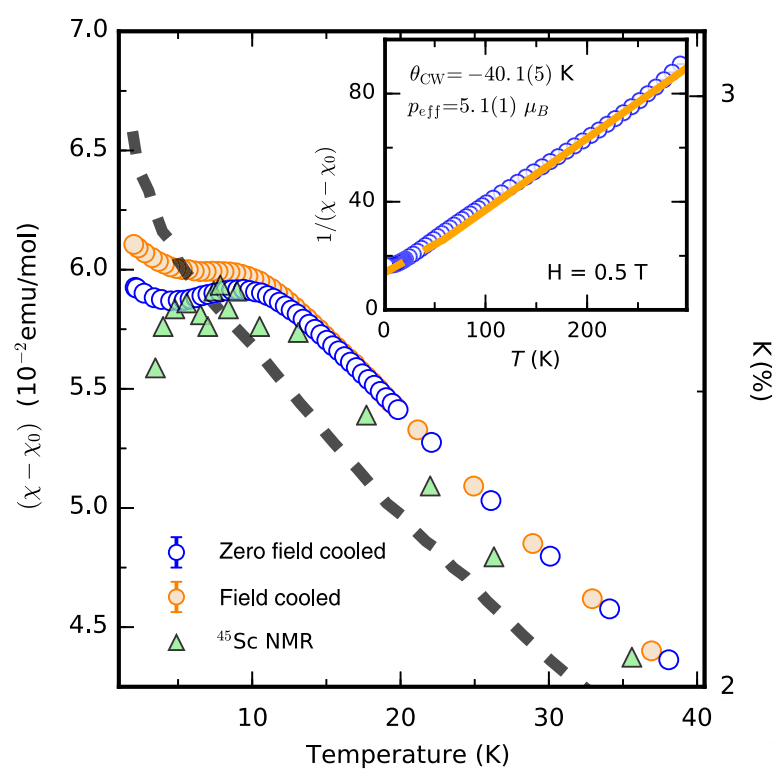

FIG. 2. Magnetic susceptibility of $\mathrm{FeSc}_{2} \mathrm{~S}_{4}$. The low- temperature susceptibility is shown in the main panel. A temperature independent contribution of $\chi_{0}=0.03 \mathrm{emu} / \mathrm{mol}$ resulting from $\leq 0.05 \%$ residual FeS has been subtracted. Open circles are the susceptibility measured on our sample, dashed line is bulk susceptibility data from Fritsch et al. [14], and triangles show the NMR Knight shift data from Ref. [15]. Inset: Inverse susceptibility, where the solid line is a linear fit to a Curie-Weiss law.

Agreement between the measured bulk susceptibility of our samples and NMR Knight shift measurements indicates an association between high chemical purity and magnetic ordering in $\mathrm{FeSc}_{2} \mathrm{~S}_{4}$.

\section{B. Specific heat}

While the $T$ dependence of the specific heat of our powder samples shown in Fig. 3 has qualitative similarities to previous reports, there are quantitative differences $[14,15]$. No sharp anomaly signifying a phase transition is visible near the $12 \mathrm{~K}$ onset temperature for the low- $T$ Bragg peaks. However, the broad maximum in $C / T$ at $8 \mathrm{~K}$ occurs at a higher temperature than the $6 \mathrm{~K}$ maximum reported in earlier studies $[14,15]$. The turnover at $8 \mathrm{~K}$ is also considerably sharper in temperature. Both of these observations are consistent with reduced disorder in our samples. Phonon contributions to the specific heat are estimated by appropriately scaling the measured specific heat of the nonmagnetic isostructural compound $\mathrm{CdIn}_{2} \mathrm{~S}_{4}$ as described in Appendix A 2. The phonon contribution, shown as a dashed line in Fig. 3(a), is subtracted from the total specific heat to yield the magnetic component. From this we infer the magnetic entropy $S_{m}$ shown in Fig. 3(b). Rather than exceeding the $R \ln (5)$ entropy available for a $S=2$ system, the magnetic entropy plateaus at approximately $70 \%$ of the available spin entropy, approaching

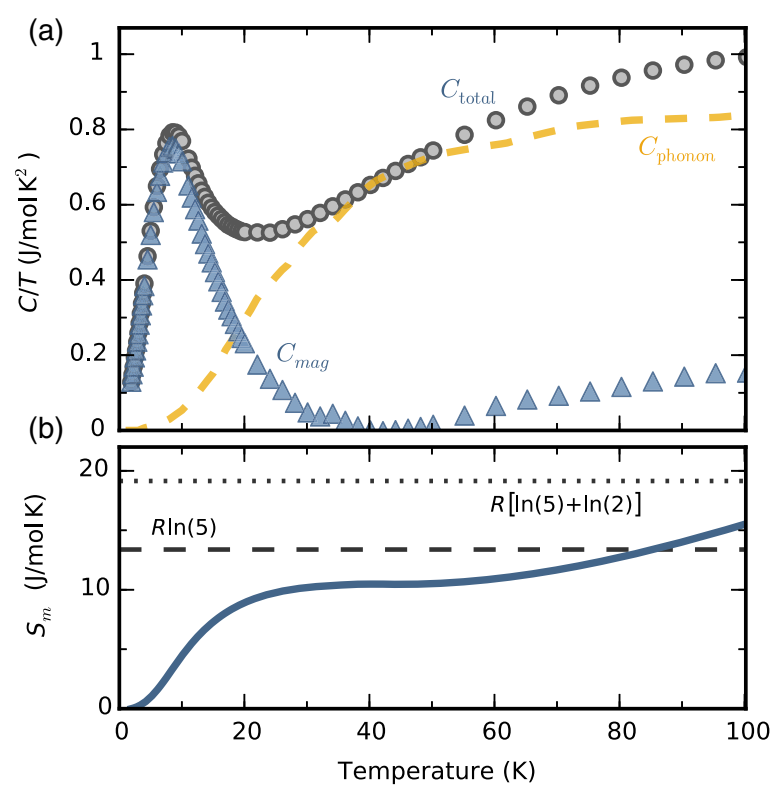

FIG. 3. Specific heat for powder samples of $\mathrm{FeSc}_{2} \mathrm{~S}_{4}$ used in this work. (a) Total specific heat (gray circles) and estimated magnetic contribution (blue triangles). Dashed yellow line is the estimated phonon contribution. (b) Estimated magnetic entropy of $\mathrm{FeSc}_{2} \mathrm{~S}_{4}$.

$R \ln (5)$ at $80 \mathrm{~K}$. Only above $80 \mathrm{~K}$ does the entropy begin to recover the additional $R \ln (2)$ associated with the twofold orbital degeneracy. Thus, our specific heat data and analysis indicates that the orbital sector in $\mathrm{FeSc}_{2} \mathrm{~S}_{4}$ is quenched for temperatures below $\sim 80 \mathrm{~K}$.

\section{Orbital degeneracy breaking}

An orbitally nondegenerate state at low temperatures is at odds with the previously accepted crystal structure for $\mathrm{FeSc}_{2} \mathrm{~S}_{4}$; we shall thus first reexamine the low-temperature crystal structure. The $T=20 \mathrm{~K}$ neutron diffraction pattern of $\mathrm{FeSc}_{2} \mathrm{~S}_{4}$ is shown in Fig. 4(a). All peaks can be indexed in the cubic $F d \overline{3} m$ space group and no peak splitting characteristic of a symmetry lowering structural distortion is apparent. Nonetheless, Rietveld refinement of our diffraction data within the $F d \overline{3} m$ space group is not satisfactory, as it yields a systematic discrepancy between measured and calculated peak intensities. Amongst the maximal subgroups of $F d \overline{3} m$, we find a significantly improved description of the data using the tetragonal $I \overline{4} m 2$ space group. Specifically, and as described more fully in Appendix B 1, the goodness of fit $\chi^{2}$ is reduced from 1.87 to 1.30 in going from $F d \overline{3} m$ to $I \overline{4} m 2$. Figure 4(b) shows the new unit cell, the resulting Rietveld refinement is shown in Fig. 4(a), and details of the refinement are presented in Table I.

Within our measurement resolution the $\mathrm{FeSc}_{2} \mathrm{~S}_{4}$ unit cell maintains its cubic metric so that any tetragonal distortion is limited to $\Delta a / a=0.002(2)$. The $I \overline{4} m 2$ unit cell contains two crystallographically distinct $\mathrm{Fe}$ sites, each forming a 
(a)

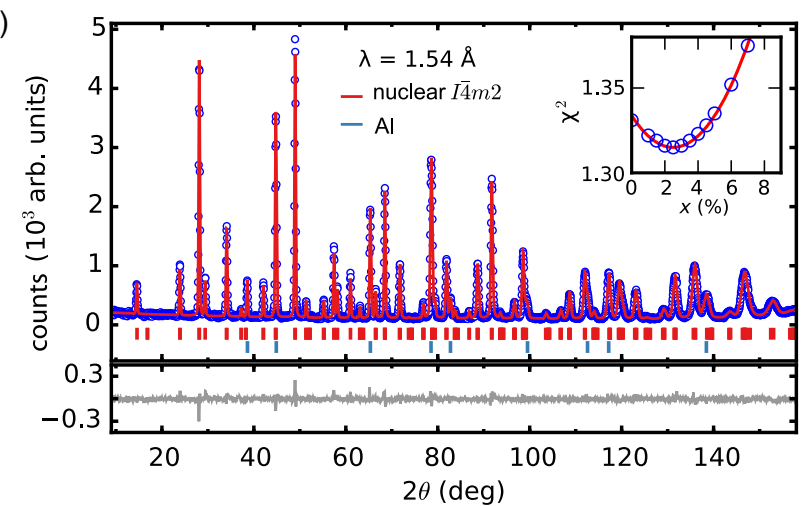

(b)

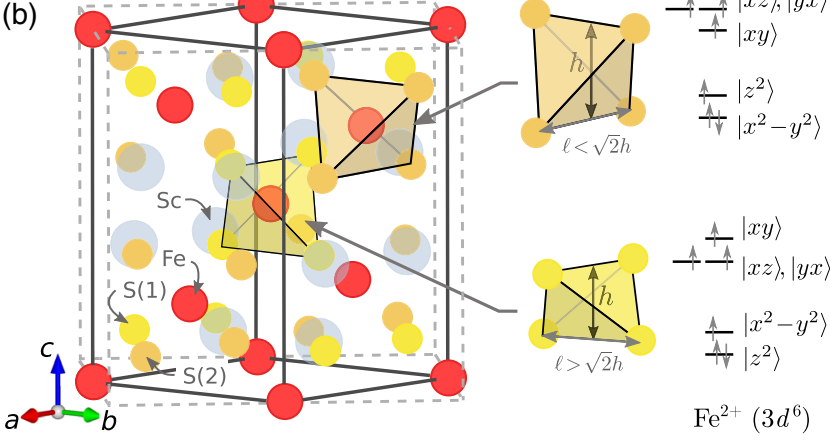

the tetrahedral symmetry around each $\mathrm{Fe}^{2+}$ site, lifting the orbital degeneracy of the Fe $e$ orbital manifold so that a hole occupies the $\left|z^{2}\right\rangle$ orbital for one sublattice and the $\left|x^{2}-y^{2}\right\rangle$ orbitals for the other. This particular distortion minimizes macroscopic strain while fully quenching the orbital moment. High-resolution x-ray diffraction measurements show that the average structure is cubic at least to $100 \mathrm{~K}$, but reveal a large anisotropic microstrain broadening of the diffraction peaks, which is consistent with an incipient structural transition (see Appendix A 1). Further ultrahigh-resolution neutron and x-ray diffraction experiments that span the relevant temperature regime where the structural phase transition may occur are under way.

While no deviations from cubic symmetry have been reported thus far, the particular low-temperature structure we report resolves a number of inconsistencies in the literature. First, Mössbauer spectroscopy consistently indicates two distinct $\mathrm{Fe}$ sites $[30,31]$. This was previously ascribed to $\mathrm{Fe}-\mathrm{Sc}$ site mixing at the level of $30 \%$ [31], which seems implausible given the disparate ionic radii and charges of $\mathrm{Fe}^{2+}$ and $\mathrm{Sc}^{3+}$. This explanation is furthermore inconsistent with our neutron diffraction data, which offer excellent contrast between $\mathrm{Fe}$ and Sc. Even at room temperature the Mössbauer spectra necessitate a second Fe site in small fractions, and below $\sim 50 \mathrm{~K}$ the quadrupolar splitting from each respective site diverges. Since the Mössbauer time scale is much slower than that of thermal fluctuations, the high-temperature quadrupolar splitting indicates a static disorder on the Fe site, possibly resulting from a local distortion nucleated around defects. Second, optical measurements in the far infrared show signatures of symmetry breaking [21,32]. Two bands around $467 \mathrm{~cm}^{-1}$ are observed in the $300 \mathrm{~K}$ absorption spectra, which are forbidden in the $F d \overline{3} m$ space group and have lacked a satisfactory explanation [32]. The extra absorption bands are consistent with a static or dynamic local distortion at room temperature.

Any signatures of a structural distortion in our diffraction data are subtle as the low-temperature structure remains metrically cubic. A small distortion is, however, generally consistent with expectations for tetrahedrally coordinated $\mathrm{Fe}^{2+}$. Indeed, any structural anomaly associated with orbital ordering in the related compound $\mathrm{FeCr}_{2} \mathrm{~S}_{4}$ is apparent only through careful line shape analysis of high-resolution x-ray synchrotron diffraction data [33]. We also see that for $\mathrm{FeSc}_{2} \mathrm{~S}_{4}$ a tetragonal distortion is essential to consistently describe both the magnetic ordering and the corresponding excitations within a spin-wave picture.

\section{Magnetic ordering}

The magnetic peaks may be indexed in the tetragonal unit cell using a single propagation vector of either $q_{m}=$ $(0,0,1)_{t}$ or $q_{m}=\left(\frac{1}{2}, \frac{1}{2}, 0\right)_{t}$, where the subscript $t$ explicitly 


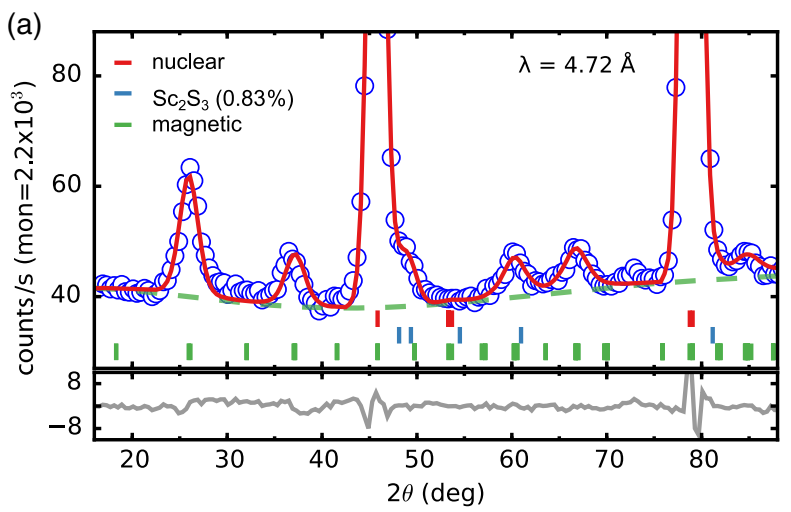

(b)

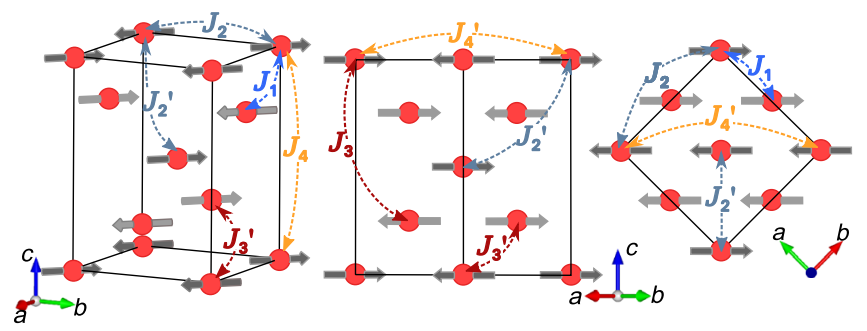

FIG. 5. (a) Neutron powder diffraction collected on MACS at $1.6 \mathrm{~K}$. Open symbols are observed intensities, solid line is the calculated intensity for the magnetic structure shown in (b), and dashed green line is background. (b) Proposed magnetic structure of $\mathrm{FeSc}_{2} \mathrm{~S}_{4}$ shown in perspective view and projected along the $(110)_{t}$, and $(001)_{t}$ directions of the tetragonal cell. Identical moments on crystallographically distinct Fe sites are drawn in light and dark gray to highlight the two CT sublattices.

indicates the tetragonal cell. The resulting magnetic structure and refinement are shown in Figs. 5(a) and 5(b). Refinements for magnetic models using a propagation vector of either $(0,0,1)_{t}$ or $\left(\frac{1}{2}, \frac{1}{2}, 0\right)_{t}$ are indistinguishable so that, with the present resolution, a unique solution cannot be determined from powder diffraction alone. However, as we discuss below, the powder-averaged inelastic magnetic neutron scattering cross section is best accounted for based on the $\left(\frac{1}{2}, \frac{1}{2}, 0\right)_{t}$ propagation vector. Various models for the magnetic ordering in $\mathrm{FeSc}_{2} \mathrm{~S}_{4}$ are discussed in Appendix B 2. The resulting magnetic structure is a collinear antiferromagnet with all moments either lying in the $a b$ plane along (110)-type directions [Fig. 5(c)] or parallel to the $c$ axis [Fig. 11(e)]. Because the $\left(\frac{1}{2}, \frac{1}{2}, 0\right)_{t^{-}}$ and $(0,0,1)_{t}$-type magnetic Bragg peaks of the centered tetragonal cell are coincident within the resolution of our measurement, the moment direction cannot be uniquely determined from the powder diffraction data. Nevertheless, refinements for each model give an identical ordered moment of $\langle m\rangle=1.76(5) \mu_{B}$ at $1.6 \mathrm{~K}$. Assuming the ordered state is homogeneous throughout the sample, this is $44(1) \%$ of the $4 \mu_{B}$ saturation magnetization for the fully localized high-spin state of $\mathrm{Fe}^{2+}$ and $g=2$. The anomalously low ordered moment is not uncommon for insulating $A$-site spinels [34,35] and may result from geometric frustration and orbital disorder [36]. Indeed, the collinear antiferromagnetic ordering we observe is a highly frustrated ground state in the CT lattice of Fig. 5. The structure satisfies only antiferromagnetic next-nearest-neighbor (NNN) exchange interactions $J_{2}$. Both the nearest-neighbor (NN) $J_{1}$ and NNN interplanar $J_{2}^{\prime}$ exchange terms compete with $J_{2}$, and cancel at the mean-field level. The result is a spin system that lowers its energy mainly through interactions within the tetragonal basal plane.

We now examine the thermal phase transition to this ordered state. Figure 6(a) shows the temperature-dependent magnetic moment obtained from Rietveld refinements of full diffraction patterns. There is a smooth increase in the ordered moment upon cooling below a critical temperature

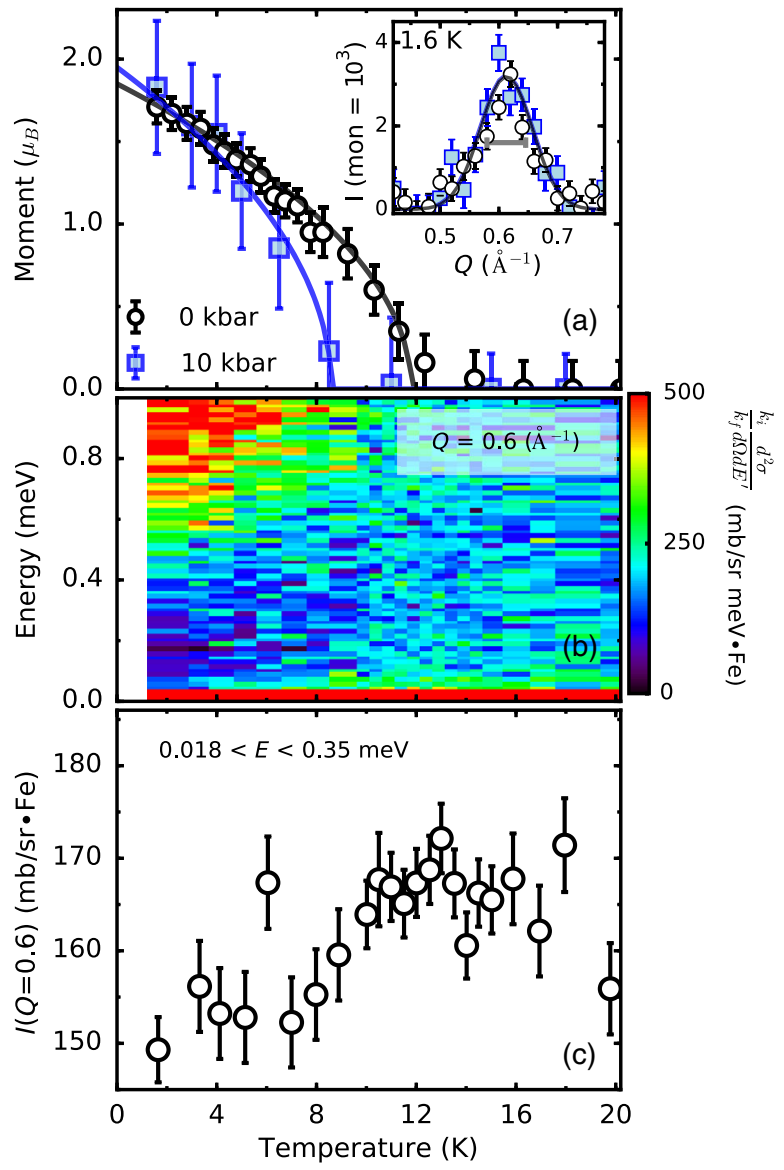

FIG. 6. (a) Temperature and pressure dependence of the ordered magnetic moment in $\mathrm{FeSc}_{2} \mathrm{~S}_{4}$, solid lines are a fit to magnetic order parameter with $\beta=0.54(4)$, saturated moment of 1.9(1) $\mu_{B}$, and $T_{c}$ of 11.8(2) and 8.6(8) $\mathrm{K}$ for 0 and $10 \mathrm{kbar}$, respectively. Inset: Background subtracted magnetic Bragg peak measured under 0 and $10 \mathrm{kbar}$ hydrostatic pressure. Horizontal line indicates instrumental resolution. Error bars represent 1 standard deviation. (b) Temperature -dependent low-energy inelastic scattering around the critical wave vector measured on CNCS integrated over $0.52<Q<0.72 \AA^{-1}$. (c) Low -energy integrated inelastic scattering around the critical wave vector integrated over $0.52<Q<0.72 \AA^{-1}$ and $0.018<E<0.35 \mathrm{meV}$. 
of $T_{c}=11.8(2) \mathrm{K}$, which at ambient pressure can be described as $M_{0}\left(1-T / T_{c}\right)^{\beta}$, with $\beta=0.54(4)$ and $M_{0}=$ 1.9(1) $\mu_{B}$. A similar temperature dependence is observed under 10-kbar hydrostatic pressure, though with a reduced transition temperature $T_{c}=8.6(8) \mathrm{K}$. The inset of Fig. 6 shows that the magnetic correlation length $[\xi=56(16) \AA]$ is unaffected by pressure to within error.

The development of magnetic order in $\mathrm{FeSc}_{2} \mathrm{~S}_{4}$ is remarkable by both the absence of critical behavior in the specific heat Fig. 3, and in the low temperature dependence of the inelastic scattering at the critical wave vector of $0.6 \AA^{-1}$, and for energy transfer $E \ll k_{b} T$ [Figs. 6(b) and 6(c)]. Rather than a sharp divergence as expected for critical scattering, only a small increase in low-energy inelastic spectral weight around $T_{c}$ resembling a truncated divergence is observed. This is followed by gradual buildup of inelastic spectral weight, concentrated around energy transfers of $\sim 1.5 \mathrm{meV}$. The mean-field-like exponent characterizing the $T$ dependence of the staggered magnetization also indicates a transition devoid of critical fluctuations. A possible explanation is that this is not a thermodynamic phase transition but instead a crossover in a finite-sized system, with the length set by the orbitally defined tetragonal domain size. In the frustrated pyrochlore $\mathrm{ZnCr}_{2} \mathrm{O}_{4}$, a first-order transition abruptly selects an ordered magnetic state out of a highly degenerate manifold of states [37]. In contrast, for $\mathrm{FeSc}_{2} \mathrm{~S}_{4}$ the structural, and in this case also orbital, phase transition occurs at a substantially higher temperature than the magnetic transition. The curious noncritical development of staggered magnetization may be understood if the orbital transition produces small domains that effectively truncate magnetic criticality and preclude an actual phase transition. A strong sensitivity of the extent of orbital ordering to sample purity might explain why this magnetic order was not previously reported: The correlation length of the orbital ordering may be shorter in samples with greater chemical disorder, as indicated by a large Curie tail in the low- $T$ susceptibility. This, in turn, could lead to a shorter magnetic correlation length, a broader magnetic crossover regime, and broader magnetic peaks that could go undetected.

\section{E. Magnetic excitations}

We now discuss magnetic excitations in $\mathrm{FeSc}_{2} \mathrm{~S}_{4}$. An overview of the normalized inelastic neutron scattering intensity $\tilde{I}(Q, E)$ is presented in Figs. 7 (a) and 7(b) for 0 and 10 kbar hydrostatic pressures, respectively. The excitation spectrum is consistent with previous reports [16] and, we see, can be qualitatively accounted for by spin-wave excitations from an ordered antiferromagnet in the tetragonal cell. There are intense, dispersive magnetic excitations emanating from $Q=0.6 \AA^{-1}$ with spectral weight extending to $\sim 5.5 \mathrm{meV}$. At low energies and in the neighborhood of the critical wave vector $\left(0.6 \AA^{-1}\right)$ the intensity continuously decreases with decreasing energy below $\sim 1 \mathrm{meV}$ to a level just above background. Based on this, one may be tempted to implicate a gap in the excitation spectrum [16]. However, for a polycrystalline sample, the inelastic neutron intensity reflects the average intensity on the Ewald sphere, which can decrease rapidly with energy for dispersive, correlated systems even though the excitations may be gapless. A gap in the excitation spectrum is signaled by the complete absence of spectral weight at low energies around the critical wave vector. In contrast, for $\mathrm{FeSc}_{2} \mathrm{~S}_{4}$ the highresolution data in Fig. 7(d) show intensity, albeit weak, down to $0.05 \mathrm{meV}$, so that any energy gap in the excitation

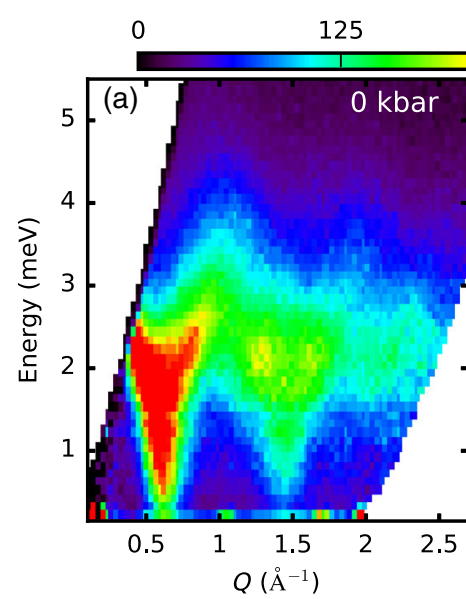
$250 \quad \frac{k_{i}}{k_{f}} \frac{d^{2} \sigma}{d \Omega d E^{\prime}}$
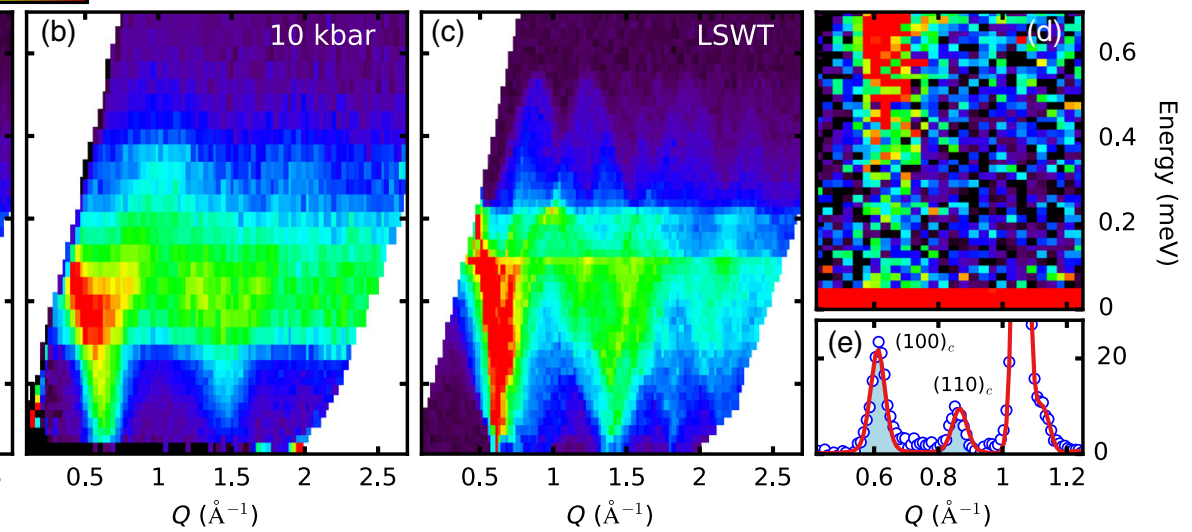

FIG. 7. (a),(b) Powder-averaged inelastic neutron scattering cross section for $\mathrm{FeSc}_{2} \mathrm{~S}_{4}$ measured at $1.6 \mathrm{~K}$ and $0 \mathrm{kbar}$ (a) and $10 \mathrm{kbar}$ (b). The cross section is placed into absolute units by calibrating against the integrated intensity from structural Bragg reflections. (c) Calculated powder-averaged neutron scattering intensity for spin waves in the collinear magnetic structure shown in Figs. 5(b) and 5(c). (d) Low-energy inelastic neutron scattering measured on CNCS. In (e) the elastic scattering is shown with magnetic diffraction peaks highlighted in light blue. Inelastic intensity is conspicuously absent near the $(110)_{c}$-type magnetic Bragg peaks, indexed in the cubic cell. 
spectrum must be smaller than this. A finite orbital domain size that limits the magnetic correlation length would also introduce a domain-size-dependent energy gap in the excitation spectrum. In this case, the low-energy spectrum would reflect a potentially inhomogeneous domain size distribution.

Although directional information is degraded by powder averaging, $\tilde{I}(Q, E)$ holds valuable information that correlates scalar lengths and energy scales. We first extract the normalized powder-averaged dynamical spin correlation function from the neutron intensity $(g / 2)^{2} \tilde{\mathcal{S}}(Q, E)=$ $1 / 2 \tilde{I}(Q, E) /\left|r_{0} f(Q)\right|^{2}$. Here, we use the dipole approximation for the $\mathrm{Fe}^{2+}\left(3 d^{6}\right)$ form factor. To determine the dominant correlation length scales independent of any model Hamiltonian, we compare the energy-integrated inelastic signal to the spherically averaged equal time structure factor,

$$
\begin{aligned}
\tilde{\mathcal{S}}(Q) & =\int_{0.2}^{6} \tilde{\mathcal{S}}(Q, E) d E \\
& =\frac{1}{3}\left(\left\langle\left(S_{0}\right)^{2}\right\rangle+\sum_{d}\left\langle S_{0} \cdot S_{d}\right\rangle \frac{\sin (Q d)}{Q d}\right),
\end{aligned}
$$

where $\left\langle S_{0} \cdot S_{d}\right\rangle$ is the average spin-spin correlator between sites separated by the distance $d$. Information regarding the magnetic bond energies may be extracted from the first moment sum rule [38,39]. For the following Heisenberg Hamiltonian including a single ion anisotropy term,

$$
\mathcal{H}=\sum_{j, j^{\prime}} J_{j j^{\prime}} \mathbf{S}_{\mathbf{j}} \cdot \mathbf{S}_{\mathbf{j}^{\prime}}+\sum_{j} D_{j}\left(S_{j}^{z}\right)^{2}
$$

the powder-averaged first moment sum rule yields

$$
\begin{aligned}
\langle E\rangle_{Q} & =\int_{0.2}^{6} E \times \tilde{\mathcal{S}}(Q, E) d E \\
& =-\frac{1}{3} \sum_{d} B_{d}\left(1-\frac{\sin (Q d)}{Q d}\right)+\mathcal{D},
\end{aligned}
$$

where $B_{d}=z J_{d}\left\langle S_{0} \cdot S_{d}\right\rangle$, is the total magnetic bond energy for a superexchange interaction $J$ across $z$ bonds at distance $d$, and the constant $\mathcal{D}=\frac{1}{N} \sum_{j} D_{j}\left[S(S+1)-3\left\langle\left(S_{j}^{z}\right)^{2}\right\rangle\right]$ is related to the sum over single-site anisotropy energies.

The measured energy-integrated inelastic intensity and first moment are shown in Figs. 8(a) and 8(b), respectively. Because of the kinematic limit for inelastic neutron scattering, the data only sample the full spectrum for $Q>0.65 \AA^{-1}$. The kinematic restrictions are especially severe for the first moment; because this quantity heavily weights higher-energy spectral weight, the first moment can be accurately extracted from our data only for $Q>0.7 \AA^{-1}$. While the static structure factor exhibits well-defined peaks distinguishing length scales that
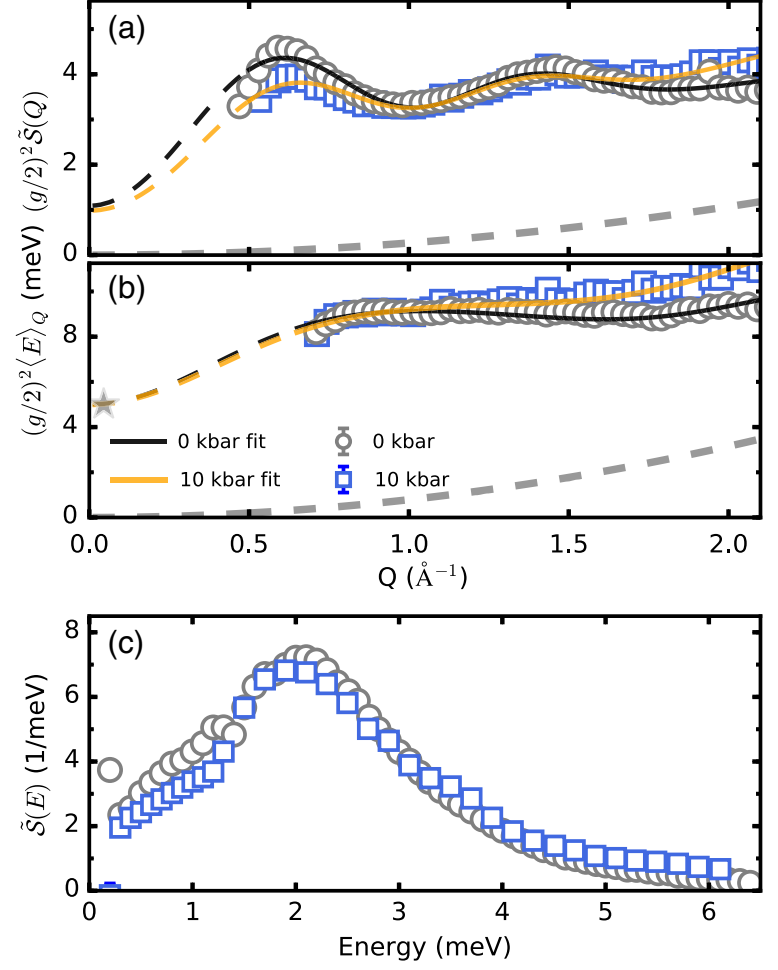

FIG. 8. (a) Static structure factor $\tilde{\mathcal{S}}(Q)$ obtained by correcting for the $\mathrm{Fe}^{2+}$ form factor and integrating the measured intensity between $0.2<E<6 \mathrm{meV}$. Solid lines are a fit to a powderaveraged structure factor as described in the text. (b) First moment of the neutron scattering intensity integrated between $0.2<E<6 \mathrm{meV}$. The gray star indicates the $Q=0$ first moment as extrapolated from terahertz measurements and the static structure factor. Solid lines are a fit to the powderaveraged first moment sum rule described in the text. Gray dashed lines in (a) and (b) are the estimated phonon contribution. (c) Momentum-integrated inelastic scattering, integrated between $0.45<Q<1.6 \AA^{-1}$. Error bars representing 1 standard deviation are smaller than the symbol size.

dominate the magnetic correlations, the first moment is relatively featureless and flat as a function of momentum transfer. A momentum-independent first moment implies the ground-state energy is dominated either by a local energy scale through $\mathcal{D}$ or by competing interactions covering a range of length scales [40]. The two contributions are clearly distinguished in the $Q=0$ limit where magnetic bond energy terms in Eq. (7) vanish. Because of the kinematic limits for inelastic neutron scattering, a fit of Eq. (7) to the neutron scattering data alone is insufficient to constrain $\mathcal{D}$. However, at $Q=0$ the first moment and static structure factor are related as follows: $\langle E\rangle_{Q=0}=\mathcal{D}=$ $\langle\hbar \omega\rangle_{0} \tilde{\mathcal{S}}(0)$, where $\langle\hbar \omega\rangle_{0}$ is the energy averaged over the fluctuation spectrum at $Q=0$. Time-domain terahertz spectroscopy has shown there to be a single well-defined mode at $\hbar \omega=4.5 \mathrm{meV}$ that dominates the dynamic susceptibility at $Q=0$ [22]. Using this result, we may apply a single-mode approximation to obtain a lower bound 
TABLE II. Exchange interactions and spin-spin correlations for distinct spin pairs in $\mathrm{FeSc}_{2} \mathrm{~S}_{4}$ extracted from fits to experimental data. Bonds are labeled according to Figs. 5(b) and 5(c). Magnetic interactions labeled $J_{i}$ and $J_{i}^{\prime}$ cannot be distinguished and so are represented by their average. The factors $(g / 2)^{2}\left\langle\left(S_{0}\right)^{2}\right\rangle=3.3(3)$ and $(g / 2)^{2} \mathcal{D}=5.0(8) \mathrm{meV}$ are also extracted from these fits at both 0 and $10 \mathrm{kbar}$.

\begin{tabular}{lrrrrrr}
\hline \hline & & \multicolumn{2}{c}{$(g / 2)^{2}\left\langle S_{0} \cdot S_{d}\right\rangle$} & & \multicolumn{2}{c}{$(g / 2)^{2} B_{d}(\mathrm{meV})$} \\
\cline { 3 - 4 } \cline { 6 - 7 } & $d(\AA)$ & $0 \mathrm{kbar}$ & $10 \mathrm{kbar}$ & & $0 \mathrm{kbar}$ & $10 \mathrm{kbar}$ \\
\hline$J_{1}$ & 4.55 & $0.1(4)$ & $-0.4(3)$ & & $-1.4(3)$ & $-1.2(6)$ \\
$J_{2}$ & 7.43 & $-2.7(2)$ & $-2.0(2)$ & & $-0.3(2)$ & $-0.2(1)$ \\
$J_{3}$ & 8.70 & $-0.6(2)$ & $-0.4(2)$ & & $\cdots$ & $\cdots$ \\
$J_{4}$ & 10.51 & $0.6(3)$ & $0.6(3)$ & & $\cdots$ & $\cdots$ \\
\hline \hline
\end{tabular}

on the anisotropy energy $\mathcal{D}=4.5 \mathrm{meV} \times \tilde{\mathcal{S}}(Q=0)$. The correspondingly constrained fits to the static structure factor and first moment are shown in Fig. 8. We allow for a $Q^{2}$-dependent background in each of the fits, which accounts for phonon contributions to the background signal. The extracted correlators and bond energies are listed in Table II.

From the static structure factor fit we find $(g / 2)^{2}\left\langle\left(S_{0}\right)^{2}\right\rangle=3.3(3)$, and $(g / 2)^{2} \mathcal{D}=5.0(8) \mathrm{meV}$ at 0 and 10 kbar. Given their similar ranges, magnetic interactions labeled $J_{i}$ and $J_{i}^{\prime}$ in Figs. 5(b) and 5(c) cannot be distinguished and so are represented by their average. Consistent with the reductions in $T_{c}$, the application of 10 kbar hydrostatic pressure appears to slightly reduce the overall magnetic correlations, although any differences in the magnetic correlators and bond energies between the 0 - and 10-kbar data are difficult to discern because of the increased background scattering from the stainless steel pressure vessel.

The sign and magnitude of spin correlators we extract are consistent with the collinear magnetic order shown in Fig. 5(a) and support a model where NNN interactions $\left(J_{2}\right)$ dominate. This analysis suggests that, at least in terms of the magnetic correlations, $\mathrm{Fe}$ spins in $\mathrm{FeSc}_{2} \mathrm{~S}_{4}$ form two interpenetrating CT sublattices, where $J_{2}$ couples spins on the same sublattice and $J_{1}$ fails to produce significant intersublattice correlations. Fits to the first-moment data converge, including only contributions from bonds $J_{1}$ and $J_{2}$ as well as $\mathcal{D}$, and we report only this minimal parameter set here. Interestingly, $\mathcal{D}$ emerges as the dominant energy scale. Such a large single-ion anisotropy scale indicates proximity of the magnetically ordered state to local singlet formation.

Figure 8(c) shows the momentum-averaged inelastic intensity that approximates the magnetic density of states. The effect of $10 \mathrm{kbar}$ hydrostatic pressure is a slight transfer of spectral weight from around $1 \mathrm{meV}$ to energies between 5 and $6 \mathrm{meV}$.

Integrating the total measured dynamical spin correlation function over the region $0.2<E<6 \mathrm{meV}$ and $0.45<Q<1.6 \AA^{-1}$, we obtain an approximation to the total inelastic spectral weight $\left\langle\delta m^{2}\right\rangle=$ $3\left(g \mu_{B}\right)^{2} \iint Q^{2} \tilde{\mathcal{S}}(Q, E) d Q d E / \int Q^{2} d Q=13(1) \mu_{B}^{2}$ at $0 \mathrm{kbar}$ and $\left\langle\delta m^{2}\right\rangle=12(1) \mu_{B}^{2}$ at $10 \mathrm{kbar}$. Adding the static (elastic) and dynamic (inelastic) contributions yields a total moment of $m_{\mathrm{tot}}^{2}=\langle m\rangle^{2}+\left\langle\delta m^{2}\right\rangle=16(3) \mu_{B}^{2}$, which is less than $g^{2} S(S+1)=24 \mu_{B}^{2}$ expected for $S=2$ and $g=2$. There are several non-mutually-exclusive explanations for this shortfall: (i) significant inelastic spectral weight exists beyond the 6-meV range of the experiment, (ii) the orbital component of the magnetism is not fully quenched, so the $g$ factor is less than the spin-only value of 2, (iii) static magnetic disorder transfers a portion of the magnetic scattering cross section to elastic diffuse magnetic scattering that is masked by strong incoherent nuclear scattering from $\operatorname{Sc}\left(\sigma_{\text {inc }}=4.5 \mathrm{~b}\right)$. The count rate expected for additional spectral weight required to satisfy the sum rule distributed over a broad $\left(10^{\circ}\right)$ scattering angle is less than 0.1 counts/s on the scale of Fig. 5(a), which is below the sensitivity of our measurements. Any or all of the above three scenarios could account for the shortfall in magnetic spectral weight.

Surprisingly, for such a large spin $(S=2)$ antiferromagnet, the inelastic scattering dominates the spectral weight, contributing over 4 times that of the static portion. For a fully spin-polarized ground state, the magnetic inelastic scattering consists entirely of spin excitations that are transverse to the ordered moment direction (spin waves) and have an available spectral weight of $g^{2} S(S+1)-$ $g^{2} S^{2}=g^{2} S \approx 8$, when $g=2$. For comparison, the observed inelastic spectral weight for $\mathrm{FeSc}_{2} \mathrm{~S}_{4}$ is $\left\langle\delta m^{2}\right\rangle=13(1)$, which implies that the Néel state in $\mathrm{FeSc}_{2} \mathrm{~S}_{4}$ is strongly renormalized by quantum fluctuations, as anticipated near a quantum phase transition. Quantum fluctuations, which reduce the ordered moment, allow for spin excitations that are longitudinal fluctuations and an associated increase of inelastic spectral weight.

\section{F. Spin-wave model}

Having identified pertinent magnetic interactions in $\mathrm{FeSc}_{2} \mathrm{~S}_{4}$, we now compare the measured excitation spectrum with a minimal effective spin Heisenberg model. While one may contest the validity of a spin-wave expansion for such a strongly renormalized state as in $\mathrm{FeSc}_{2} \mathrm{~S}_{4}$ with $\left\langle(\delta m)^{2}\right\rangle=13>g^{2} S=8$, the semiclassical description might provide a reasonable account of longwavelength dispersive transverse components of the magnetic excitation spectrum. Figures 7(d) and 7(e) juxtapose the low-energy inelastic neutron scattering signal with the elastic signal in the neighborhood of the first two magnetic Bragg peaks, indexed in the cubic unit cell as $(100)_{c}$ and $(110)_{c}$, respectively. A comparison of the elastic and inelastic intensities in this region immediately reveals an important clue that proves essential to determining the 
magnetic order in $\mathrm{FeSc}_{2} \mathrm{~S}_{4}$. While there is strong elastic scattering around both $(100)_{c}$ and $(110)_{c}$, inelastic scattering is found only around $(100)_{c}\left(0.6 \AA^{-1}\right)$. Since $(100)_{c}$ and $(110)_{c}$ are equivalent by symmetry, the energy eigenvalues of magnetic excitations must be identical at these wave vectors. The absence of powder-averaged inelastic scattering near $(110)_{c}$ must then reflect different matrix elements for $Q=0.6 \AA^{-1}$ and $0.84 \AA^{-1}$. In the low-energy long-wavelength limit the inelastic magnetic intensity $\tilde{I}(Q, E)$ scales with the elastic scattering except for the polarization factor, which extinguishes scattering associated with spin components along wave vector transfer $\mathbf{Q}$. For magnetic structures that are consistent with the observed diffraction pattern in the cubic cell, the polarization factor can at most extinguish only half of the spectral weight at $(110)_{c}$ compared to $(100)_{c}$. The suppression observed experimentally is, however, more than a factor of 4 , so that indexing of the magnetic structure with a cubic unit cell must be rejected. It is this feature of the inelastic scattering that identifies the magnetic wave vector $q_{m}=$ $\left(\frac{1}{2}, \frac{1}{2}, 0\right)_{t}$ and the magnetic order shown in Fig. 5. With $q_{m}=\left(\frac{1}{2}, \frac{1}{2}, 0\right)_{t}$, the magnetic Bragg peaks at 0.6 and $0.84 \AA^{-1}$ correspond to distinct points in the Brillouin zone, $\left(\frac{1}{2}, \frac{1}{2}, 0\right)_{t}$ and $\left(\frac{1}{2}, \frac{1}{2}, 1\right)_{t}$, that can have different excitation spectra. While this conclusion does not rely on a spin-wave expansion, we now show that spin waves in a $q_{m}=\left(\frac{1}{2}, \frac{1}{2}, 0\right)_{t}$ structure can account for these unusual low-energy features of $\mathrm{FeSc}_{2} \mathrm{~S}_{4}$, and indeed they account for the full inelastic magnetic neutron scattering cross section.

Within the tetragonal structure there are at least seven magnetic exchange pathways identified in Figs. 5(b) and 5(c) that must be considered in a simple spin-wave model. Additionally, the two $\mathrm{Fe}^{2+}$ sites in the unit cell have distinct tetragonal environments, permitting two distinct single-ion anisotropy terms in the magnetic Hamiltonian. Dzyaloshinksii exchange terms are also symmetry allowed for all spin pairs in the tetragonal cell. The powderaveraged inelastic magnetic neutron scattering cross section was computed within linear spin-wave theory (LSWT) as implemented in the SpinW program [41] for the minimal Hamiltonian in Eq. (6). For simplicity, we include only Heisenberg exchange terms, which are labeled in Figs. 5(b) and 5(c) and listed in Table II, plus distinct single-ion easyplane anisotropy terms on the two distinct $\mathrm{Fe}^{2+}$ sites. Guided by the static structure factor and first moment sum rule analysis, we adjust the exchange constants to obtain the best qualitative agreement between the measured and calculated spectra. The results of this calculation are shown in Fig. 7(c) for the exchange interactions detailed in Table II and single-ion easy-plane anisotropy energies $D_{a b}=0.10(5) \mathrm{meV}$ and $D_{a b}^{\prime}=0.05(4) \mathrm{meV}$. We may combine the anisotropy energies with the measured total and ordered moments to gives $(g / 2)^{2} \mathcal{D}_{\mathrm{SW}}=4(2) \mathrm{meV}$. To
TABLE III. Renormalized exchange interactions used in the linear spin-wave model in units of $\mathrm{meV}$, bonds are labeled according to Fig. 5. The values reported here are renormalized by the reduced ordered moment $Z=\langle m\rangle / g S=1.76 / 4$.

\begin{tabular}{lcccccc}
\hline \hline$J_{1}$ & $J_{2}$ & $J_{2}^{\prime}$ & $J_{3}$ & $J_{3}^{\prime}$ & $J_{4}$ & $J_{4}^{\prime}$ \\
\hline$-0.14(8)$ & $0.7(1)$ & $0.6(6)$ & $0.02(2)$ & $0.02(2)$ & $-0.09(7)$ & $-0.09(7)$ \\
\hline \hline
\end{tabular}

aid meaningful comparison of the LSWT-derived exchange constants and magnetic bond energies in the strongly fluctuating Néel state of $\mathrm{FeSc}_{2} \mathrm{~S}_{4}$, LSWT exchange constants in Table III have been renormalized by the reduced ordered moment $\langle m\rangle / g S=1.76 / 4$. It is encouraging that the magnitudes of these exchange parameters are generally consistent with band structure calculations for $\mathrm{FeSc}_{2} \mathrm{~S}_{4}$, which give $J_{1}=0.01 \mathrm{meV}$ and $J_{2}=0.37 \mathrm{meV}$ [42].

While the powder-averaged neutron scattering data do not contain sufficient information to constrain the seven exchange and two anisotropy parameters, the purpose of the spin-wave calculation we present here is to show that the character particularly of low-energy spin excitations in $\mathrm{FeSc}_{2} \mathrm{~S}_{4}$ can be accounted for with the proposed $q_{m}=$ $\left(\frac{1}{2}, \frac{1}{2}, 0\right)_{t}$ wave vector within space group $I \overline{4} m 2$. Indeed, the spin-wave model captures the general intensity distribution including the concentration of high-energy spectral weight around $Q \approx 1 \AA^{-1}$ and the inelastic intensity differences around the $\left(\frac{1}{2}, \frac{1}{2}, 0\right)_{t}$ and $\left(\frac{1}{2}, \frac{1}{2}, 1\right)_{t}$ magnetic reflections. In fact, the pattern of inelastic magnetic scattering is quite restrictive and appears to be consistent with only a magnetic propagation vector $q_{m}=\left(\frac{1}{2}, \frac{1}{2}, 0\right)_{t}$ in the tetragonal cell and the particular magnetic ordering pattern shown in Fig. 5(b). To make this point, a comparison of the calculated linear spin wave neutron intensity for different models of magnetic ordering in $\mathrm{FeSc}_{2} \mathrm{~S}_{4}$ is presented in Appendix B 3. The weak scattering intensity around $\left(\frac{1}{2}, \frac{1}{2}, 1\right)_{t}$ is a consequence of the highly frustrated nature of the magnetic ordering in the tetragonal cell. For the collinear ordering, the dominant exchange interactions, which have a component along the $c$ axis $\left(J_{2}^{\prime}\right.$ and $\left.J_{1}\right)$, are frustrated and cancel at the mean-field level. The result is dimensional reduction through frustration. A consequence of this frustration is softening of magnetic excitations around $\left(\frac{1}{2}, \frac{1}{2}, 1\right)_{t}$, which manifests as weak diffuse scattering intensity near $0.85 \AA^{-1}$ in the powder-averaged spectrum.

Comparison with the spin-wave expansion yields further important insight. The inclusion of easy-plane anisotropy terms are essential to stabilize the magnetic structure and for agreement between model and data. These terms gap only transverse excitations which are spin fluctuations out of the $a-b$ plane. At the level of linear spin-wave theory, there is always a gapless transverse fluctuation within the $a-b$ plane. Thus, inasmuch as the spin-wave expansion accurately accounts for long-wavelength 
magnetic excitations in $\mathrm{FeSc}_{2} \mathrm{~S}_{4}$, the data are consistent with a gapless excitation spectrum.

While the spin-wave model appears to capture most features of the excitation spectrum, we do not claim it to be unique. Furthermore, it is not clear that a spin-wave expansion should be relevant for the highly renormalized Néel state of $\mathrm{FeSc}_{2} \mathrm{~S}_{4}$. Discrepancies of the spin-wave model are particularly apparent at energies, above $2 \mathrm{meV}$, where longitudinal and multimagnon excitations that are not accounted for in the $1 / S$ expansion may be expected to play an important role. The spectral weight available for two-magnon (longitudinal) scattering $\left\langle\delta m_{2 M}^{2}\right\rangle=\Delta S(\Delta S+1)$ is directly related to the reduction in sublattice magnetization, $\langle m\rangle^{2}=(S-\Delta S)^{2}$, and $\Delta S=S-\left\langle S_{z}\right\rangle$. Assuming the moment reduction is entirely a consequence of quantum fluctuations, the total fraction of spectral weight observed that results from non-spin-wave excitations is $\Delta S(\Delta S+1) / S(S+1)=2.391 / 6=40 \%$.

\section{DISCUSSION}

Our measurements show that the putative spin-orbital liquid $\mathrm{FeSc}_{2} \mathrm{~S}_{4}$, in fact, has a magnetically ordered ground state. Furthermore, we present evidence that this order arises from a centered tetragonal structure with two crystallographically distinct low-symmetry sites. Static and dynamic magnetic correlations can be understood only within the tetragonal unit cell with a magnetic propagation vector $q_{m}=\left(\frac{1}{2}, \frac{1}{2}, 0\right)_{t}$. Although the chemical unit cell remains metrically cubic, the alternating compression and expansion of the coordinating sulfur tetrahedra lift the orbital degeneracy of the ideal tetrahedral coordination. In concordance with these results, the weak $Q$ dependence of the first moment is a clear indication of the corresponding anisotropic spin Hamiltonian.

The nature of the thermal transition leading to this strongly fluctuating Néel state is rather peculiar. It is already apparent from the absence of a sharp specific heat anomaly that the magnetic ordering transition is not a phase transition in the thermodynamic limit but a crossover in a finite-sized system. The relevant length scale of this system may be the 53(6) A correlation length that we detect for the magnetic ordering. An obvious scenario to consider but one that we shall ultimately reject is that both this finite correlation length and the reduced staggered magnetization are a result of chemical inhomogeneity in the sample. The effect of such inhomogeneity would be to nucleate small volume fractions of magnetic order around chemical impurities or structural defects. The diamond lattice antiferromagnet is known to be particularly sensitive to defect-induced magnetism through the order-by-quencheddisorder mechanism [43]. Further, since the neutron signal is sensitive only to the product of the ordered moment and volume fraction of the ordered phase, diffraction cannot distinguish between a homogeneous state with a small ordered moment and a finite correlation length and an inhomogeneous state with a large ordered moment.

However, the fact that the inelastic magnetic scattering can be described by spin waves in an ordered, renormalized, antiferromagnet favors a homogeneous picture. Synthesized with special attention to eliminating residual oxygen contamination, our samples furthermore appear cleaner than those typically reported in literature where magnetic ordering has not been reported. Structural refinements at $100 \mathrm{~K}$ definitively exclude any nonstoichiometries beyond a $\sim 1 \%$ limit of detection, and also tightly constrain any Fe:Sc mixing to 3(1)\% (See Appendix A 1). From the antisite defect concentration we may extract an average spacing between site inversion defects of 17(2) A, roughly one-third of the magnetic correlation length we measure, so that the magnetic correlations appear to be limited by some other effect.

Comparison of thermodynamic measurements on our samples and those of previous reports indicate that the defect concentration in the present samples is lower than in previous studies. The low-temperature maximum of our measured specific heat both occurs at a higher temperature and is sharper in temperature than previously reported [14]. Bulk magnetic susceptibility of our powder samples shows a turnover at $\sim 10 \mathrm{~K}$ closely resembling the local susceptibility obtained from NMR Knight shift measurements $[15,29]$. This indicates a reduced concentration of magnetic impurities or orphan spins in our samples, as these lead to a low-temperature Curie tail that was observed in previous reports though not in our sample [14,15].

The magnetic order we report here is a stable characteristic of a new generation of samples. In particular, we observe the magnetic Bragg reflections in three different samples each having undergone a different thermal processing schedule. While the maximum temperature during synthesis differs by as much as $400{ }^{\circ} \mathrm{C}$ between samples, the relative integrated intensity of the magnetic $(100)_{c}$ and nuclear $(111)_{c}$ Bragg reflections varies only between 0.17 and 0.2 across all measurements. Rather than being set by any chemical inhomogeneity, we propose that the relevant length scale for the magnetic order in $\mathrm{FeSc}_{2} \mathrm{~S}_{4}$ is the orbital domain size established at a highertemperature first-order cubic-to-tetragonal transition that lifts the orbital degeneracy.

The ordering phenomenon we report for $\mathrm{FeSc}_{2} \mathrm{~S}_{4}$ has similarities with another $A$-site magnetic spinel $\mathrm{CoAl}_{2} \mathrm{O}_{4}$ [34], where neutron scattering measurements on single crystals show signatures of antiferromagnetic domains developing across an arrested first-order, order-by-disorder, magnetic transition [13]. Magnetic domain formation in $\mathrm{CoAl}_{2} \mathrm{O}_{4}$ is, however, proposed to be a result of geometric frustration [13]. In $\mathrm{FeSc}_{2} \mathrm{~S}_{4}$, the weak tetragonal distortion we observe points towards another mechanism for disorder and domain formation: in the tetragonal cell there is an alternating compression and elongation of the $\mathrm{Fe}$ 
coordinating $\mathrm{S}$ tetrahedron, though the diffraction measurement does not uniquely identify which iron site has the compressed and which has the elongated tetrahedral environment. If the tetrahedral distortion is not coherent between many unit cells, the result will be static orbital disorder and concomitant exchange disorder. There would also be disorder in the distribution of single-ion anisotropy terms. Since the tetragonal distortion associated with this structural transition is so small $(\Delta a / a<0.2 \%)$ a significant density of such quenched disorder that would limit the magnetic correlation length is plausible. Monte Carlo simulations of the order-by-disorder transition in the face-centered-cubic lattice show that magnetic domains engender thermally excited defects and domain walls that reduce the sublattice magnetization [44]. Hence, the presence of magnetic domains may account for the missing magnetic spectral weight and the finite magnetic correlation length in $\mathrm{FeSc}_{2} \mathrm{~S}_{4}$.

It is apparent that in $\mathrm{FeSc}_{2} \mathrm{~S}_{4}$ many energy scales, including crystal field splitting, spin-orbit coupling, and frustrated magnetic exchange interactions compete and conspire to produce very rich physics. The $0.2 \%$ upper bound we place on a tetragonal distortion in $\mathrm{FeSc}_{2} \mathrm{~S}_{4}$ admits proximity to spin-orbital quantum criticality. In the mean-field phase diagram proposed by Chen et al. [18], the critical point occurs at $x_{c}=\left(J_{2} / \lambda\right)=\frac{1}{16}$. Using the experimentally determined value for the spin-orbit coupling constant $\lambda=1.57(25) \mathrm{meV}$ [22] and the unrenormalized magnetic exchange $J_{2}$ used in the spin-wave model, we estimate that $\left(J_{2} / \lambda\right)>0.20$, which places $\mathrm{FeSc}_{2} \mathrm{~S}_{4}$ within the magnetic and orbitally ordered regime. While our detection of magnetic Bragg peaks corresponding to spin order with a $57 \AA$ correlation length would appear to put an end to the idea that $\mathrm{FeSc}_{2} \mathrm{~S}_{4}$ is a spin-orbital liquid, the strongly enhanced fluctuations we observe are a direct manifestation of proximity to a critical point. The dominant single-ion anisotropy energy scale we extract is a further indication of proximity to a local singlet phase. This implies it may be possible to drive $\mathrm{FeSc}_{2} \mathrm{~S}_{4}$ through the quantum phase transition and stabilize the spin-orbital liquid ground state by application of a suitable perturbation $[19,23]$. We find a reduced staggered magnetization and shift of spectral weight towards higher energies under a hydrostatic pressure of $10 \mathrm{kbar}$. This suggests we should look to hydrostatic pressure beyond $10 \mathrm{kbar}$ for access to the spin-orbital quantum critical point in $\mathrm{FeSc}_{2} \mathrm{~S}_{4}$. Magnetic order, on the other hand, might be enhanced through uniaxial stress or annealing of the cubic-totetragonal phase transition.

\section{ACKNOWLEDGMENTS}

We would like to thank N. P. Armitage and N. J. Laurita for helpful discussions and comments on this manuscript. We also acknowledge Juscelino Leao for assistance with the high-pressure sample environment and Nicholas Butch for providing transmission measurements of the stainless steel pressure vessel. Work at IQM was supported by the U.S. Department of Energy, Office of Basic Energy Sciences, Division of Material Sciences and Engineering under Grant No. DE-FG02-08ER46544. Work at ORNL was sponsored by the Division of Scientific User Facilities, Office of Basic Energy Science, U.S. Department of Energy (DOE). Work at NIST utilized facilities supported in part by the National Science Foundation under Agreement No. DMR-1508249. Use of the Advanced Photon Source at Argonne National Laboratory was supported by the U.S. Department of Energy, Office of Science, Office of Basic Energy Sciences, under Contract No. DE-AC02-06CH11357.

\section{APPENDIX A: SAMPLE CHARACTERIZATION}

\section{Synchrotron powder diffraction}

The $T=100 \mathrm{~K}$ synchrotron powder diffraction data pattern collected on 11-BM and the corresponding Rietveld refinement is shown in Fig. 9. All diffraction peaks are indexed within the cubic $F d \overline{3} m$ unit cell and we find a good refinement to the $100 \mathrm{~K}$ diffraction data using the reported structure for $\mathrm{FeSc}_{2} \mathrm{~S}_{4}$. However, significant microstrain broadening of the diffraction peaks is apparent in the peak shapes. An isotropic strain broadening in the cubic cell is accounted for in GSAS utilizing the semiempirical Stephens peak shape [45] with cubic strains of $S_{400}=0.19 \%, S_{200}=0.01 \%$. The large difference between the two symmetry allowed strain parameters is consistent with an incipient structural transition. Our x-ray powder

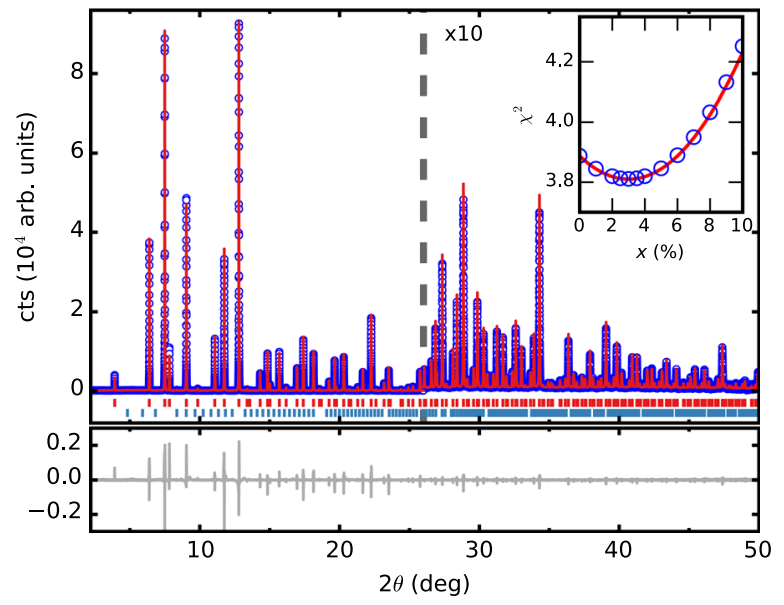

FIG. 9. Rietveld analysis of high-resolution synchrotron x-ray data collected on 11-BM at $100 \mathrm{~K}$. Blue circles are measured data, red line is the calculated diffraction pattern, and red vertical bars indicate the positions of nuclear reflections in the $F d \overline{3} m$ space group. There is also a contribution from a $0.15 \% \mathrm{Sc}_{2} \mathrm{O}_{3}$ contamination phase. The difference between calculated and measured data is shown in the lower panel. Inset: Goodnessof-fit parameter $\left(\chi^{2}\right)$ versus the Fe-Sc antisite inversion. The $\mathrm{Sc}_{2} \mathrm{O}_{3}$ contamination phase is not included in refinements used to determine the site inversion. 
TABLE IV. Atomic parameters obtained from Reitveld refinements of synchrotron x-ray diffraction data at $100 \mathrm{~K}$ for $\mathrm{FeSc}_{2} \mathrm{~S}_{4}$ samples used in this work. The space group is $\bar{F} \bar{d} 32$ (227) and the lattice parameter is refined to $a=10.51115(1) \AA$. Reitveld refinements result in $\chi^{2}=2.83$ and $R_{\text {Bragg }}=5.59 \%$.

\begin{tabular}{lccccrc}
\hline \hline Atom & Site & $x$ & \multicolumn{1}{c}{$y$} & $z$ & Occ & \multicolumn{1}{c}{$B_{\text {iso }}$} \\
\hline $\mathrm{Fe}$ & $8 \mathrm{a}$ & 0.125 & 0.125 & 0.125 & 0.94 & $0.313(2)$ \\
$\mathrm{Sc}$ & $8 \mathrm{a}$ & 0.125 & 0.125 & 0.125 & 0.06 & $0.311(2)$ \\
$\mathrm{Fe}$ & $16 \mathrm{~d}$ & 0.500 & 0.500 & 0.500 & 0.03 & $0.313(2)$ \\
$\mathrm{Sc}$ & $16 \mathrm{~d}$ & 0.500 & 0.500 & 0.500 & 0.97 & $0.311(2)$ \\
$\mathrm{S}$ & $32 \mathrm{e}$ & $0.2556(1)$ & $0.2556(1)$ & $0.2556(1)$ & 1.00 & $0.352(2)$ \\
\hline \hline
\end{tabular}

diffraction data additionally show the presence of a minor $(0.15 \%)$ contamination phase of $\mathrm{Sc}_{2} \mathrm{O}_{3}$ in our sample, below the limit of detection in our neutron diffraction measurements.

The magnetic properties of $A$-site spinel compounds are highly sensitive to the degree of chemical disorder resulting from inversion between atomic species occupying the $A$ and $B$ sites of the spinel structure [34,46]. To quantify any inversion present in our sample, we refine out synchrotron data allowing for antisite disorder as described by $\left(\mathrm{Fe}_{1-x} \mathrm{Sc}_{x}\right)\left[\mathrm{Sc}_{2-x} \mathrm{Fe}_{x}\right] \mathrm{S}_{4}$. The inset of Fig. 9 shows the goodness-of-fit parameter versus the site inversion $x$, which indicates a small amount of site inversion $x=0.03(1)$ in our sample. The site inversion is further constrained by refinement of neutron powder diffraction data described in Appendix B 1. The value of $\chi^{2}$ in the inset of Fig. 9 is larger than reported for the full Rietveld refinement because only a single phase was included in Rietveld refinements used to estimate the site inversion. We have checked that the inclusion of the contamination phase does not affect the conclusion of the site-mixing analysis. Rietveld refinement parameters are listed in Table IV.

\section{Phonon contribution to specific heat}

Phonon contributions to the specific heat of $\mathrm{FeSc}_{2} \mathrm{~S}_{4}$ are estimated by scaling the measured specific heat of the nonmagnetic analog spinel compound $\mathrm{CdIn}_{2} \mathrm{~S}_{4}$. The measured specific heat for $\mathrm{CdIn}_{2} \mathrm{~S}_{4}$ is extracted from Ref. [14]. To estimate the phonon contribution to the specific heat of $\mathrm{FeSc}_{2} \mathrm{~S}_{4}$, this must be scaled by the relative Debye temperatures $\Theta$ of the two compounds,

$$
\frac{\Theta_{\mathrm{CdIn}_{2} \mathrm{~S}_{4}}^{3}}{\Theta_{\mathrm{FeSc}_{2} \mathrm{~S}_{4}}^{3}}=\left(\frac{m_{\mathrm{Fe}}+2 m_{\mathrm{Sc}}+4 m_{\mathrm{S}}}{m_{\mathrm{Cd}}+2 m_{\mathrm{In}}+4 m_{\mathrm{S}}}\right)^{3 / 2},
$$

where $m_{x}$ is the atomic mass of element $x$. The rescaled temperature for the phonon specific heat is then given by

$$
T_{\mathrm{FeSc}_{2} \mathrm{~S}_{4}}=\frac{\Theta_{\mathrm{CdIn}_{2} \mathrm{~S}_{4}}}{\Theta_{\mathrm{FeSc}_{2} \mathrm{~S}_{4}}} T_{\mathrm{CdIn}_{2} \mathrm{~S}_{4}},
$$

and the rescaled lattice contribution is given by

$$
C_{\mathrm{FeSc}_{2} \mathrm{~S}_{4}}=\frac{\Theta_{\mathrm{CdIn}_{2} \mathrm{~S}_{4}}^{3}}{\Theta_{\mathrm{FeSc}_{2} \mathrm{~S}_{4}}^{3}} C_{\mathrm{CdIn}_{2} \mathrm{~S}_{4}}
$$

The scaled phonon contribution to the specific heat of $\mathrm{FeSc}_{2} \mathrm{~S}_{4}$ is shown as a red dashed line in Fig. 3.

\section{APPENDIX B: STRUCTURAL AND MAGNETIC MODELS}

\section{Low-temperature structural refinements}

Neutron powder diffraction measurements collected on BT-1 at $20 \mathrm{~K}$ are shown in Fig. 10. The four panels display identical diffraction data but with Rietveld refinements corresponding to different structural models for $\mathrm{FeSc}_{2} \mathrm{~S}_{4}$. Details of each refinement are listed in Table V. Note that $\mathrm{Fe}:$ Sc site mixing is not included in these refinements, but we check that the conclusions are not affected by this.

The tetragonal lattice parameter $a_{t}$ is simply related to the cubic cell by $a_{t}=a_{c} / \sqrt{2}$. Refinements for each structural model show that the lattice remains metrically cubic in that $\left(a_{c}-c\right) / a_{c}<0.2 \%$. While there are no obvious qualitative differences between the refinements for each structural model, close quantitative comparison reveals a significant improvement in the quality of fit for the tetragonal unit cell and space group $I \overline{4} m 2$.

\section{Candidate magnetic structures}

Irreducible representations and their basis vectors for each crystal symmetry and magnetic ordering wave vector in $\mathrm{FeSc}_{2} \mathrm{~S}_{4}$ are calculated using the SARAh program [28]. Here, we outline each of the candidate magnetic structures consistent with the neutron diffraction data in the parent cubic $F d \overline{3} m$ unit cell and the proposed tetragonal $I \overline{4} m 2$ cell.

In the cubic spinel structure all magnetic peaks are indexed with a propagation vector of $\mathbf{k}=(001)$. The decomposition of the magnetic representation for the $\mathrm{Fe}$ site $(0.125,0.125,0.125)$ is $\Gamma_{\text {mag }}=\Gamma_{1}^{2}+\Gamma_{2}^{2}+2 \Gamma_{4}^{2}$, and the irreducible representations and associated basis vectors are detailed in Table VI. Satisfactory magnetic refinements in the $F d \overline{3} m$ structural space group are only possible including one equally weighted basis vector from each of the two irreducible representations $\Gamma_{1}$ and $\Gamma_{2}$ : either $\psi_{1}$ and $\psi_{3}$ or $\psi_{2}$ and $\psi_{4}$. The two structures are simply related by a $\pi / 2$ rotation of all spins and are indistinguishable in powder diffraction data. The resulting magnetic structure and refinements are shown in Fig. 11(a).

For the tetragonal $I \overline{4} \mathrm{~m} 2$ cell the magnetic Bragg peaks may all be indexed with magnetic propagation vectors $\mathbf{k}_{\mathbf{1}}=$ $(001)$ or $\mathbf{k}_{\mathbf{2}}=\left(\frac{1}{2} \frac{1}{2} 0\right)$. There are two Fe sites in the primitive basis. For $\mathrm{Fe}_{1}$ at $(0,0,0)$ the decomposition of the magnetic representation is $\Gamma_{\mathrm{mag}}=\Gamma_{2}^{1}+\Gamma_{5}^{2}$ for $\mathbf{k}_{\mathbf{1}}$ and $\Gamma_{\mathrm{mag}}=\Gamma_{2}^{1}+$ $\Gamma_{3}^{1}+\Gamma_{4}^{1}$ for $\mathbf{k}_{2}$. For $\mathrm{Fe}_{2}$ at $(0,0.5,0.25)$, the decomposition of the magnetic representation is $\Gamma_{\mathrm{mag}}=\Gamma_{4}^{1}+\Gamma_{5}^{2}$ for $\mathbf{k}_{\mathbf{1}}$ 

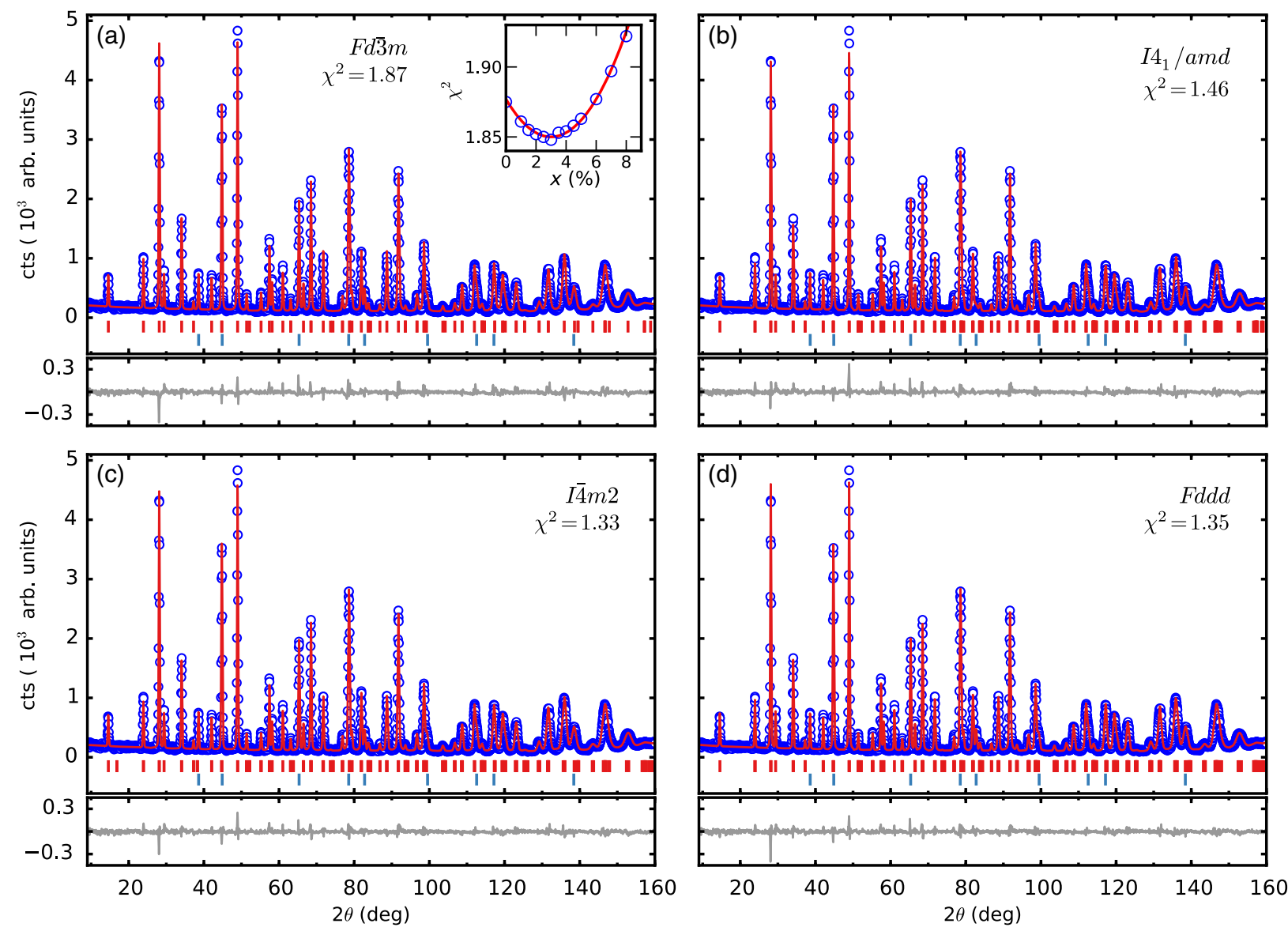

FIG. 10. Structural models for $\mathrm{FeSc}_{2} \mathrm{~S}_{4}$. (a)-(d) Rietveld analysis of neutron powder diffraction measured on BT-1 at 20 K. Blue circles are measured data, red line is the calculated diffraction pattern, and red vertical bars indicate the positions of nuclear reflections in the respective space group. Contributions from the aluminum sample environment are indicated by blue vertical bars. The difference between calculated and measured data is shown in the lower panel for (a)-(d). Inset in (a) shows the goodness-of-fit parameter $\left(\chi^{2}\right)$ versus the $\mathrm{Fe}-\mathrm{Sc}$ antisite inversion in the cubic model.

and $\Gamma_{\text {mag }}=\Gamma_{1}^{1}+\Gamma_{2}^{1}+\Gamma_{4}^{1}$ for $\mathbf{k}_{1}$. Irreducible representations (Irrep.) and associated basis vectors for the propagation vectors $\mathbf{k}_{\mathbf{1}}$ and $\mathbf{k}_{\mathbf{2}}$ are detailed in Tables VII, VIII, and IX, X, respectively.

The refined magnetic structure for $\mathbf{k}_{\mathbf{1}}=(001)$ is shown in Fig. 11(c) and is identical to that in Fig. 11(a) when transformed into the cubic cell. This structure is described with the irreducible representation $\Gamma_{5}$ on both Fe sites and equally weighted basis vectors.

TABLE V. Candidate crystal structures for $\mathrm{FeSc}_{2} \mathrm{~S}_{4}$ at $20 \mathrm{~K}$.

\begin{tabular}{lcccc}
\hline \hline Symmtery & Cubic & \multicolumn{4}{c}{ Tetragonal } & Tetragonal & Orthorhombic \\
Space group & $F d \overline{3} m$ & $I 4_{1} /$ amd & $I \overline{4} m 2$ & $F d d d$ \\
\hline$a(\AA)$ & $10.507(1)$ & $7.437(1)$ & $7.434(1)$ & $10.514(1)$ \\
$b(\AA)$ & $\ldots$ & $\ldots$ & $\ldots$ & $10.514(1)$ \\
$c(\AA)$ & $\ldots$ & $10.495(1)$ & $10.493(1)$ & $10.491(1)$ \\
$c / a_{c}$ & 1 & 0.998 & 0.998 & 0.998 \\
$\chi^{2}$ & 1.87 & 1.46 & 1.33 & 1.35 \\
$R_{\text {wp }}(\%)$ & 7.92 & 6.99 & 6.68 & 6.71 \\
$R_{\text {Bragg }}(\%)$ & 6.73 & 5.57 & 5.25 & 5.13 \\
\hline \hline
\end{tabular}

When the magnetic peaks are indexed with $\mathbf{k}_{\mathbf{1}}=\left(\frac{1}{2} \frac{1}{2} 0\right)$ many equally good solutions are permitted by the data. In Figs. 11(e) and 11(d), we show the magnetic structure and refinement for $\Gamma_{4}$ on $\mathrm{Fe}_{1}$ and $\Gamma_{2}$ on $\mathrm{Fe}_{2}$.

TABLE VI. Basis vectors for the space group $F d \overline{3} m$ with $\mathbf{k}=(001)$

\begin{tabular}{|c|c|c|c|c|c|}
\hline Irrep. & Basis vector & Atom & $m_{\| a}$ & $m_{\| b}$ & $m_{\| c}$ \\
\hline \multirow[t]{4}{*}{$\Gamma_{1}$} & \multirow[t]{2}{*}{$\psi_{1}$} & 1 & 0 & 4 & 0 \\
\hline & & 2 & -4 & 0 & 0 \\
\hline & \multirow[t]{2}{*}{$\psi_{2}$} & 1 & 4 & 0 & 0 \\
\hline & & 2 & 0 & -4 & 0 \\
\hline \multirow[t]{4}{*}{$\Gamma_{2}$} & \multirow[t]{2}{*}{$\psi_{3}$} & 1 & 4 & 0 & 0 \\
\hline & & 2 & 0 & 4 & 0 \\
\hline & \multirow[t]{2}{*}{$\psi_{4}$} & 1 & 0 & -4 & 0 \\
\hline & & 2 & -4 & 0 & 0 \\
\hline \multirow[t]{4}{*}{$\Gamma_{4}$} & \multirow[t]{2}{*}{$\psi_{5}$} & 1 & 0 & 0 & 8 \\
\hline & & 2 & 0 & 0 & 0 \\
\hline & \multirow[t]{2}{*}{$\psi_{6}$} & 1 & 0 & 0 & 0 \\
\hline & & 2 & 0 & 0 & -8 \\
\hline
\end{tabular}


(a)

Model 1: $F d \overline{3} m(227) \mathbf{k}=\left(\begin{array}{lll}0 & 0 & 1\end{array}\right)$
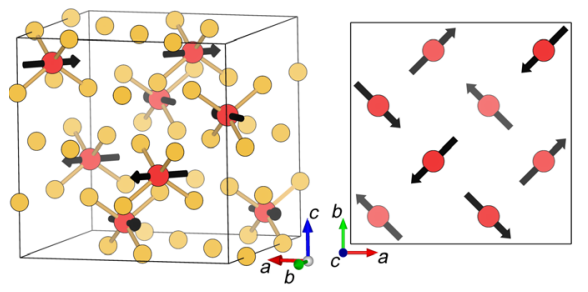

(b)

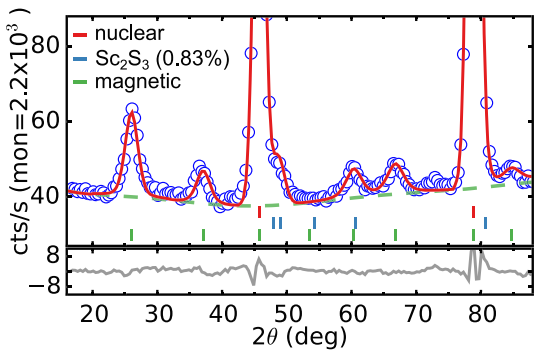

(c)

Model 2: $/ \overline{4} m 2(119) \mathbf{k}=\left(\begin{array}{lll}0 & 0 & 1\end{array}\right)$

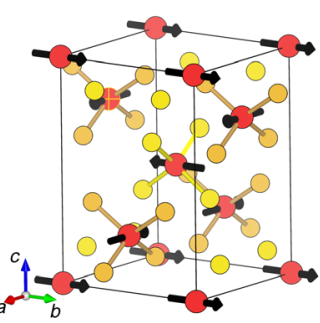

(d)

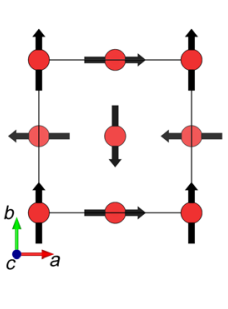

(f)

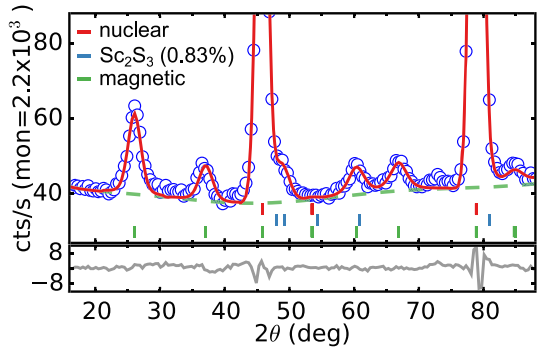

(e)
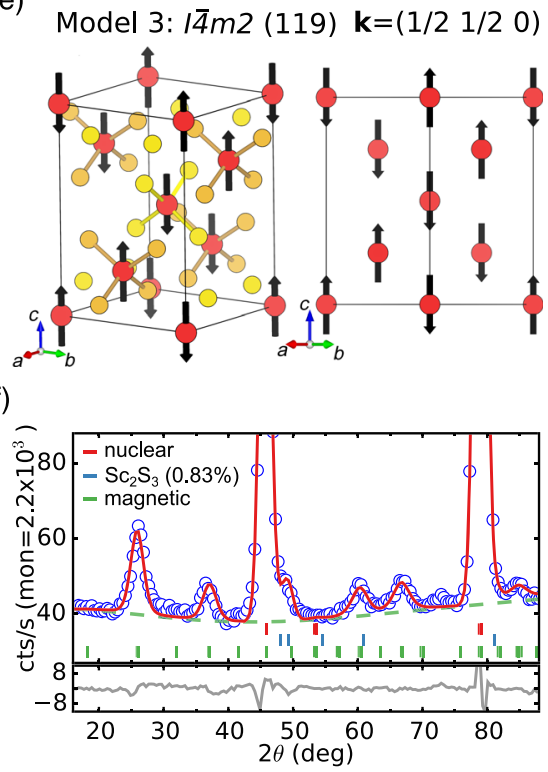

FIG. 11. Candidate magnetic structures in $\mathrm{FeSc}_{2} \mathrm{~S}_{4}$. (a),(b) Magnetic structure and corresponding Rietveld refinement for the $F d \overline{3} m$ structural space group and magnetic peaks indexed with a $\mathbf{k}=(001)$ propagation vector. (c),(d) Magnetic structure and corresponding Rietveld refinement for the $I \overline{4} m 2$ structural space group and magnetic peaks indexed with a $\mathbf{k}=(0,0,1)$ propagation vector. The resulting magnetic structure is identical to that in (a) when transformed to the cubic cell. (e),(f) Alternative magnetic structure and corresponding Rietveld refinement for the $I \overline{4} m 2$ structural space group and magnetic peaks indexed with a $\mathbf{k}=(1 / 2,1 / 2,0)$ propagation vector.
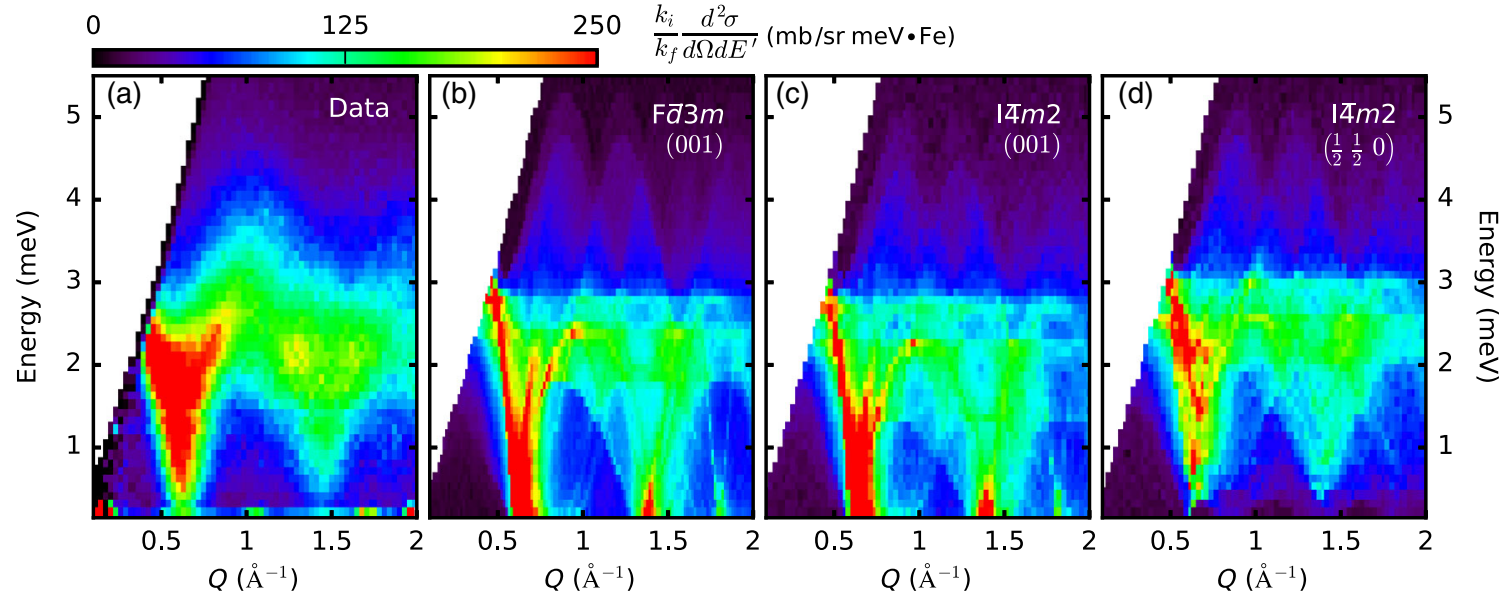

FIG. 12. Spin-wave excitations in candidate magnetic structures for $\mathrm{FeSc}_{2} \mathrm{~S}_{4}$. (a) Measured powder-averaged inelastic neutron scattering spectra at $1.6 \mathrm{~K}$ and ambient pressure. (b)-(d) Calculated powder-averaged neutron scattering intensity from spin-wave excitations for possible magnetic structures in $\mathrm{FeSc}_{2} \mathrm{~S}_{4}$. Heisenberg exchange interactions for each model are listed in Table XI.

The collinear magnetic structure shown in Figs. 5(b) and 5(d) includes irreducible representations $\Gamma_{3}$ on $\mathrm{Fe}_{1}$ and $\Gamma_{1}$ on $\mathrm{Fe}_{2}$.

\section{Magnetic excitations and spin-wave models}

The powder-averaged magnetic excitation spectra is computed for each magnetic candidate structure in $\mathrm{FeSc}_{2} \mathrm{~S}_{4}$ within linear spin-wave theory as implemented in the SpinW program [41]. For each model, the inelastic magnetic neutron scattering intensity from spin waves is calculated using a simple model Hamiltonian, including only Heisenberg-type exchange terms labeled in Fig. 5(b) as well as single-ion anisotropy terms on each $\mathrm{Fe}^{2+}$. For the cubic structure, a symmetry-disallowed easy-plane anisotropy term is included to stabilize the magnetic structure, while the tetragonal structures allow for two distinct single-ion anisotropy terms, one per crystallographic Fe site. These are included as easyplan anisotropies for models 2 and 4, and an easy-axis 
TABLE VII. Basis vectors for the space group $I \overline{4} m 2$ with $\mathbf{k}=(001)$ for for the Fe site $(0,0,0)$.

\begin{tabular}{lccccc}
\hline \hline Irrep. & Basis vector & Atom & $m_{\| a}$ & $m_{\| b}$ & $m_{\| c}$ \\
\hline$\Gamma_{2}$ & $\psi_{1}$ & 1 & 0 & 0 & 8 \\
$\Gamma_{5}$ & $\psi_{2}$ & 1 & 0 & 4 & 0 \\
& $\psi_{3}$ & 1 & 4 & 0 & 0 \\
\hline \hline
\end{tabular}

TABLE VIII. Basis vectors for the space group $I \overline{4} m 2$ with $\mathbf{k}=(001)$ for the Fe site $(0,0.5,0.25)$.

\begin{tabular}{lccccc}
\hline \hline Irrep. & Basis vector & Atom & $m_{\| a}$ & $m_{\| b}$ & $m_{\| c}$ \\
\hline$\Gamma_{4}$ & $\psi_{1}$ & 1 & 0 & 0 & 8 \\
$\Gamma_{5}$ & $\psi_{2}$ & 1 & 0 & 4 & 0 \\
& $\psi_{3}$ & 1 & -4 & 0 & 0 \\
\hline \hline
\end{tabular}

TABLE IX. Basis vectors for the space group $\mathrm{I} \overline{4} m 2$ with $\mathbf{k}=\left(\frac{1}{2} \frac{1}{2} 0\right)$ for the Fe site $(0,0,0)$.

\begin{tabular}{lccccc}
\hline \hline Irrep. & Basis vector & Atom & $m_{\| a}$ & $m_{\| b}$ & $m_{\| c}$ \\
\hline$\Gamma_{2}$ & $\psi_{1}$ & 1 & 2 & -2 & 0 \\
$\Gamma_{3}$ & $\psi_{2}$ & 1 & 2 & 2 & 0 \\
$\Gamma_{4}$ & $\psi_{3}$ & 1 & 0 & 0 & 4 \\
\hline \hline
\end{tabular}

TABLE X. Basis vectors for the space group $I \overline{4} m 2$ with $\mathbf{k}=\left(\frac{1}{2} \frac{1}{2} 0\right)$ for the Fe site $(0,0.5,0.25)$.

\begin{tabular}{lccccc}
\hline \hline Irrep. & Basis vector & Atom & $m_{\| a}$ & $m_{\| b}$ & $m_{\| c}$ \\
\hline$\Gamma_{1}$ & $\psi_{1}$ & 1 & 2 & 2 & 0 \\
$\Gamma_{2}$ & $\psi_{2}$ & 1 & 0 & 0 & 4 \\
$\Gamma_{4}$ & $\psi_{3}$ & 1 & 2 & -2 & 0 \\
\hline \hline
\end{tabular}

anisotropy for model 3. Models 1-3 are shown in Fig. 11 and model 4 is presented in the main text. Guided by the relative strength of spin correlators and magnetic bond energies extracted from the static structure factor and first moment sum rule analysis, the magnetic exchange interactions are chosen to provide the best qualitative match of the data with the simplest combination of exchange terms. Exchange constants used for each respective spin-wave calculation are detailed in Table XI. The calculated powder-averaged neutron scattering intensity for spin-wave excitations in each model are shown in Fig. 12.

The ground-state energy of each magnetic structure is as follows:

model 1: $E_{1}=0 J_{1}-8 J_{2}-4 J_{4}$,

model 2: $E_{2}=2 \times\left(0 J_{1}+4 J_{2}-8 J_{2}^{\prime}-2 J_{4}\right)$,

model 3: $E_{3}=2 \times\left(0 J_{1}-4 J_{2}+0 J_{2}^{\prime}-2 J_{4}\right)$.
TABLE XI. Unrenormalized exchange interactions used for model spin-wave calculations in $\mathrm{FeSc}_{2} \mathrm{~S}_{4}$. Bonds are labeled according to Fig. 5(b).

\begin{tabular}{lcrr}
\hline \hline & Model 1 & Model 2 & \multicolumn{1}{c}{ Model 3 } \\
\hline$J_{1}(\mathrm{meV})$ & $0.10(9)$ & $0.10(9)$ & $-0.06(4)$ \\
$J_{2}(\mathrm{meV})$ & $0.27(5)$ & $0.27(5)$ & $0.31(6)$ \\
$J_{2}^{\prime}(\mathrm{meV})$ & $\ldots$ & $0.27(5)$ & $0.27(6)$ \\
$J_{3}(\mathrm{meV})$ & $0.00(1)$ & $0.00(2)$ & $0.01(1)$ \\
$J_{3}^{\prime}(\mathrm{meV})$ & $\ldots$ & $0.00(2)$ & $0.01(1)$ \\
$J_{4}(\mathrm{meV})$ & $0.00(1)$ & $-0.01(2)$ & $-0.04(3)$ \\
$J_{4}^{\prime}(\mathrm{meV})$ & $\ldots$ & $-0.01(2)$ & $-0.04(3)$ \\
$D(\mathrm{meV})$ & $0.1(1)$ & $0.15(6)$ & $0.15(6)$ \\
$D^{\prime}(\mathrm{meV})$ & $\cdots$ & $0.05(4)$ & $0.05(4)$ \\
\hline \hline
\end{tabular}

Ground-state energies of models 3 and 4 are identical. For these models, Heisenberg NN $\left(J_{1}\right)$ and out-of-plane NNN $\left(J_{2}^{\prime}\right)$ exchange terms cancel at the mean-field level and the system is effectively a two-dimensional Heisenberg square lattice. For models 1 and 2, the NN spins are orthogonal, so the Heisenberg terms vanish.

[1] P. A. Lee, An End to the Drought of Quantum Spin Liquids, Science 321, 1306 (2008).

[2] L. Balents, Spin Liquids in Frustrated Magnets, Nature (London) 464, 199 (2010).

[3] L. F. Feiner, A. M. Oleś, and J. Zaanen, Quantum Melting of Magnetic Order due to Orbital Fluctuations, Phys. Rev. Lett. 78, 2799 (1997).

[4] Y. Kitaoka, T. Kobayashi, A. Kda, H. Wakabayashi, Y. Niino, H. Yamakage, S. Taguchi, K. Amaya, K. Yamaura, M. Takano, A. Hirano, and R. Kanno, Orbital Frustration and Resonating Valence Bond State in the Spin-1/2 Triangular Lattice $\mathrm{LiNiO}_{2}$, J. Phys. Soc. Jpn. 67, 3703 (1998).

[5] B. Keimer, D. Casa, A. Ivanov, J. W. Lynn, M. v. Zimmermann, J. P. Hill, D. Gibbs, Y. Taguchi, and Y. Tokura, Spin Dynamics and Orbital State in $\mathrm{LaTiO}_{3}$, Phys. Rev. Lett. 85, 3946 (2000).

[6] G. Khaliullin and S. Maekawa, Orbital Liquid in ThreeDimensional Mott Insulator: $\mathrm{LaTiO}_{3}$, Phys. Rev. Lett. 85, 3950 (2000).

[7] S. Nakatsuji, K. Kuga, K. Kimura, R. Satake, N. Katayama, E. Nishibori, H. Sawa, R. Ishii, M. Hagiwara, F. Bridges, T. U. Ito, W. Higemoto, Y. Karaki, M. Halim, A. A. Nugroho, J. A. Rodriguez-Rivera, M. A. Green, and C. Broholm, Spin-Orbital Short-Range Order on a Honeycomb-Based Lattice, Science 336, 559 (2012).

[8] P. Corboz, M. Lajkó, A. M. Läuchli, K. Penc, and F. Mila, Spin-Orbital Quantum Liquid on the Honeycomb Lattice, Phys. Rev. X 2, 041013 (2012).

[9] A. Krimmel, M. Mücksch, V. Tsurkan, M. M. Koza, H. Mutka, C. Ritter, D. V. Sheptyakov, S. Horn, and A. Loidl, Magnetic Ordering and Spin Excitations in the Frustrated Magnet $\mathrm{MnSc}_{2} \mathrm{~S}_{4}$, Phys. Rev. B 73, 014413 (2006). 
[10] D. Bergman, J. Alicea, E. Gull, S. Trebst, and L. Balents, Order-by-Disorder and Spiral Spin-Liquid in Frustrated Diamond-Lattice Antiferromagnets, Nat. Phys. 3, 487 (2007).

[11] J.-S. Bernier, M. J. Lawler, and Y. Baek Kim, Quantum Order by Disorder in Frustrated Diamond Lattice Antiferromagnets, Phys. Rev. Lett. 101, 047201 (2008).

[12] A. Krimmel, H. Mutka, M. M. Koza, V. Tsurkan, and A. Loidl, Spin Excitations in Frustrated A-Site Spinels Investigated with Inelastic Neutron Scattering, Phys. Rev. B 79, 134406 (2009).

[13] G. J. MacDougall, D. Gout, J. L. Zarestky, G. Ehlers, A. Podlesnyak, M. A. McGuire, D. Mandrus, and S. E. Nagler, Kinetically Inhibited Order in a Diamond-Lattice Antiferromagnet, Proc. Natl. Acad. Sci. U.S.A. 108, 15693 (2011).

[14] V. Fritsch, J. Hemberger, N. Büttgen, E.-W. Scheidt, H.-A. Krug von Nidda, A. Loidl, and V. Tsurkan, Spin and Orbital Frustration in $\mathrm{MnSc}_{2} \mathrm{~S}_{4}$ and $\mathrm{FeSc}_{2} \mathrm{~S}_{4}$, Phys. Rev. Lett. 92, 116401 (2004).

[15] N. Büttgen, J. Hemberger, V. Fritsch, A. Krimmel, M. Mücksch, H.-A. Krug von Nidda, P. Lunkenheimer, R. Fichtl, V. Tsurkan, and A. Loidl, Orbital Physics in Sulfur Spinels: Ordered, Liquid and Glassy Ground States, New J. Phys. 6, 191 (2004).

[16] A. Krimmel, M. Mücksch, V. Tsurkan, M. M. Koza, H. Mutka, and A. Loidl, Vibronic and Magnetic Excitations in the Spin-Orbital Liquid State of $\mathrm{FeSc}_{2} \mathrm{~S}_{4}$, Phys. Rev. Lett. 94, 237402 (2005).

[17] K. Kugel and D. Khomskii, The Jahn-Teller Effect and Magnetism: Transition Metal Compounds, Sov. Phys. Usp. 25, 231 (1982).

[18] G. Chen, L. Balents, and A. P. Schnyder, Spin-Orbital Singlet and Quantum Critical Point on the Diamond Lattice: $\mathrm{FeSc}_{2} \mathrm{~S}_{4}$, Phys. Rev. Lett. 102, 096406 (2009).

[19] G. Chen, A. P. Schnyder, and L. Balents, Excitation Spectrum and Magnetic Field Effects in a Quantum Critical Spin-Orbital System: The Case of $\mathrm{FeSc}_{2} \mathrm{~S}_{4}$, Phys. Rev. B 80, 224409 (2009).

[20] A. Biffin, Ch. Rüegg, J. Embs, T. Guidi, A. Loidl, V. Tsurkan, and R. Coldea, Magneic Field Dependence of Excitations Near Spin-Orbital Quantum Criticality, arXiv: 1606.09482 .

[21] L. Mittelstädt, M. Schmidt, Zhe Wang, F. Mayr, V. Tsurkan, P. Lunkenheimer, D. Ish, L. Balents, J. Deisenhofer, and A. Loidl, Spin-Orbiton and Quantum Criticality in $\mathrm{FeSc}_{2} \mathrm{~S}_{4}$, Phys. Rev. B 91, 125112 (2015).

[22] N. J. Laurita, J. Deisenhofer, L. D. Pan, C. M. Morris, M. Schmidt, M. Johnsson, V. Tsurkan, A. Loidl, and N. P. Armitage, Singlet-Triplet Excitations and Long-Range Entanglement in the Spin-Orbital Liquid Candidate $\mathrm{FeSc}_{2} \mathrm{~S}_{4}$, Phys. Rev. Lett. 114, 207201 (2015).

[23] D. Ish and L. Balents, Theory of Excitations and Dielectric Response at a Spin-Orbital Quantum Critical Point, Phys. Rev. B 92, 094413 (2015).

[24] J. R. Morey, K. W. Plumb, C. M. Pasco, B. A. Trump, T. M. McQueen, and S. M. Koopayeh, Growth and Characterization of Iron Scandium Sulfide, J. Cryst. Growth 454, 128 (2016).

[25] C. Stock, W. J. L. Buyers, R. Liang, D. Peets, Z. Tun, D. Bonn, W. N. Hardy, and R. J. Birgeneau, Dynamic Stripes and Resonance in the Superconducting and Normal Phases of $\mathrm{YBa}_{2} \mathrm{Cu}_{3} \mathrm{O}_{6.5}$ Ortho-II Superconductor, Phys. Rev. B 69 , 014502 (2004).

[26] G. Ehlers, A. A. Podlesnyak, J. L. Niedziela, E. B. Iverson, and P. E. Sokol, The New Cold Neutron Chopper Spectrometer at the Spallation Neutron Source: Design and Performance, Rev. Sci. Instrum. 82, 085108 (2011).

[27] S.-H. Lee, C. Broholm, M. F. Collins, L. Heller, A. P. Ramirez, Ch. Kloc, E. Bucher, R. W. Erwin, and N. Lacevic, Less than 50\% Sublattice Polarization in an Insulating $S=\frac{3}{2}$ Kagomé Antiferromagnet at $T \approx 0$, Phys. Rev. B 56, 8091 (1997).

[28] A. S Wills, A New Protocol for the Determination of Magnetic Structures Using Simulated Annealing and Representational Analysis (SARAh), Physica (Amsterdam) 276278B, 680 (2000).

[29] N. Büttgen, A. Zymara, C. Kegler, V. Tsurkan, and A. Loidl, Spin and Orbital Frustration in $\mathrm{FeSc}_{2} \mathrm{~S}_{4}$ Probed by ${ }^{45} \mathrm{Sc}$ NMR, Phys. Rev. B 73, 132409 (2006).

[30] L. Brossard, H. Oudet, and P. Gibart, Distribution of Strains at the $\mathrm{Fe}^{2+}$ A-Site in the Thiospinel $\mathrm{FeSc}_{2} \mathrm{~S}_{4}$, J. Phys. Colloques 37, C6-23 (1976).

[31] B. S. Son, S. J. Kim, Y. Jo, M.-H. Jung, B. W. Lee, and C. S. Kim, Magnetic Ordering in the Frustrated System $\mathrm{FeSc}_{2} \mathrm{~S}_{4}$, J. Magn. Magn. Mater. 320, e699 (2008).

[32] S. Reil, H.-J. Stork, and H. Haeuseler, Structural Investigations of the Compounds $\mathrm{ASc}_{2} \mathrm{~S}_{4}(A=\mathrm{Mn}, \mathrm{Fe}, \mathrm{Cd}), \mathrm{J}$. Alloys Compd. 334, 92 (2002).

[33] V. Tsurkan, O. Zaharko, F. Schrettle, Ch. Kant, J. Deisenhofer, H.-A. Krug von Nidda, V. Felea, P. Lemmens, J. R. Groza, D. V. Quach, F. Gozzo, and A. Loidl, Structural Anomalies and the Orbital Ground State in $\mathrm{FeCr}_{2} \mathrm{~S}_{4}$, Phys. Rev. B 81, 184426 (2010).

[34] B. Roy, A. Pandey, Q. Zhang, T. W. Heitmann, D. Vaknin, D. C. Johnston, and Y. Furukawa, Experimental Evidence of a Collinear Antiferromagnetic Ordering in the Frustrated $\mathrm{CoAl}_{2} \mathrm{O}_{4}$ Spinel, Phys. Rev. B 88, 174415 (2013).

[35] H. S. Nair, Z. Fu, J. Voigt, Y. Su, and Th. Brückel, Approaching the True Ground State of Frustrated A-Site Spinels: A Combined Magnetization and Polarized Neutron Scattering Study, Phys. Rev. B 89, 174431 (2014).

[36] S. B. Lee and L. Balents, Theory of the Ordered Phase in A-Site Antiferromagnetic Spinels, Phys. Rev. B 78, 144417 (2008).

[37] S.-H. Lee, C. Broholm, T. H. Kim, W. Ratcliff, and S-W. Cheong, Local Spin Resonance and Spin-Peierls-like Phase Transition in a Geometrically Frustrated Antiferromagnet, Phys. Rev. Lett. 84, 3718 (2000).

[38] P. C. Hohenberg and W. F. Brinkman, Sum Rules for the Frequency Spectrum of Linear Magnetic Chains, Phys. Rev. B 10, 128 (1974).

[39] M. B. Stone, I. Zaliznyak, D. H. Reich, and C. Broholm, Frustration-Induced Two-Dimensional Quantum Disordered Phase in Piperazinium Hexachlorodicuprate, Phys. Rev. B 64, 144405 (2001).

[40] M. B. Stone, Y. Chen, J. Rittner, H. Yardimci, D. H. Reich, C. Broholm, D. V. Ferraris, and T. Lectka, Frustrated ThreeDimensional Quantum Spin Liquid in $\mathrm{CuHpCl}$, Phys. Rev. B 65, 064423 (2002). 
[41] S. Toth and B. Lake, Linear Spin Wave Theory for Single- $Q$ Incommensurate Magnetic Structures, J. Phys. Condens. Matter 27, 166002 (2015).

[42] S. Sarkar, T. Maitra, R. Valentí, and T. Saha-Dasgupta, Comparative Study of $\mathrm{FeCr}_{2} \mathrm{~S}_{4}$ and $\mathrm{FeSc}_{2} \mathrm{~S}_{4}$ : Spinels with Orbitally Active a Site, Phys. Rev. B 82, 041105 (2010).

[43] L. Savary, E. Gull, S. Trebst, J. Alicea, D. Bergman, and L. Balents, Impurity Effects in Highly Frustrated DiamondLattice Antiferromagnets, Phys. Rev. B 84, 064438 (2011).
[44] M. V. Gvozdikova and M. E. Zhitomirsky, A Monte Carlo Study of the First-Order Transition in a Heisenberg FCC Antiferromagnet, JETP Lett. 81, 236 (2005).

[45] P. W. Stephens, Phenomenological Model of Anisotropic Peak Broadening in Powder Diffraction, J. Appl. Crystallogr. 32, 281 (1999).

[46] K. Hanashima, Y. Kodama, D. Akahoshi, C. Kanadani, and T. Saito, Spin Glass Order by Antisite Disorder in the Highly Frustrated Spinel Oxide $\mathrm{CoAl}_{2} \mathrm{O}_{4}$, J. Phys. Soc. Jpn. 82, 024702 (2013). 